\title{
Aorto-subclavioplastia com preservação do fluxo arterial: padronização técnica
}

\author{
José Teles de MENDONÇA*, Marcos Ramos CARVALHO*, Rika Kakuda da COSTA*, Edson FRANCO \\ FILHO*, Geodete Batista COSTA* $^{*}$
}

MENDONÇA, J.T.; CARVALHO, M.R.; COSTA, R.K.; FRANCO FILHO, E.; COSTA, G.B. - Aorto-subclavioplastia com preservação do fluxo arterial para o membro superior esquerdo. Rev. Bras. Cir. Cardiovasc., 8(2):63-82, 1993.

RESUMO: Entre fevereiro de 1984 e março de 1992, 10 pacientes portadores de coarctação da aorta foram operados utilizando-se a aorto-subclavioplastia com preservação do fluxo arterial para o membro superior esquerdo. Oito eram do sexo masculino e 2 do sexo feminino. A idade variou de 2 meses a 25 anos (média 6,3 anos) e peso de $3,1 \mathrm{~kg}$ a $60 \mathrm{~kg}$ (média $21,2 \mathrm{~kg}$ ). A técnica consistiu basicamente na desinserção da artéria subclávia esquerda e posterior reimplante, em forma de telhado, um pouco abaixo, sobre a regiăo coarcatada, produzindo uma importante ampliação da aorta preservando o fluxo arterial para o braço. $\mathrm{Na}$ realização deste procedimento deve-se atentar para dois detalhes fundamentais: a) ampla dissecção da aorta e artéria subclávia para permitir grande mobilização destes vasos; b) a abertura na parede anterior da aorta deve ser a maior possivel, de modo que a artéria subclávia, quando reimplantada, ultrapasse folgadamente a região estreitada, produzindo uma restauração anatômica da luz arterial sem grandes tensões na linha de sutura. Duas modificações na técnica original foram utilizadas: a) ressecção de um segmento da parede anterior da aorta conjuntamente com a artéria subclávia empregada em pacientes com istmo bem formado, promove uma boa correção com menor deslocamento dessa artéria; b) ressecção do segmento coarctado, anastomose término-terminal posterior e ampliação anterior com a artéria subclávia - (indicada nas crianças menores, com objetivo de eliminar, total ou parcialmente, o tecido ductal). A técnica padrăo foi utilizada em 3 pacientes, com segmento da aorta em 2, e a ressecçāo do segmento coarctado em 5 . Um paciente faleceu na operação (óbito não relacionado com a técnica) e 9 foram acompanhados (tempo minimo 12 meses, máximo 8 anos e média 3,9 anos). Todos se mantiveram assintomáticos, com pressāo arterial e intensidade normal de pulso nos membros inferiores. O estudo angiográfico pós-operatório realizado entre o segundo dia e o sexto ano (média 1,6 anos) evidenciou uma excelente ampliação da aorta com preservação do fluxo para o membro superior esquerdo em 8 pacientes. Em apenas 1 foi observado pequeno estrangulamento circular causando gradiente de $20 \mathrm{mmHg}$. Em conclusão, a aorto-subclavioplastia com preservação do fluxo arterial (técnica-padrão ou associada) assegura uma correção anatomicamente adequada, permite crescimento da aorta e pode ser empregada na grande maioria das coarctações tratadas na idade pediátrica.

DESCRITORES: aorto-subclavioplastia; aorta, coartação, cirurgia.

\section{INTRODUÇÃO}

A coarctação da aorta é um estreitamento congênito que ocorre usualmente logo após a emergên- cia da artéria subclávia esquerda, nas adjacências da inserção do canal arterial. Incide em, aproximadamente, um de cada 12000 nascimentos e representa $8 \%-10 \%$ de todas as cardiopatias congênitas.

Trabalho realizado na Unidade Cardiotorácica de Sergipe, Hospital de Clínicas Dr.Augusto Leite, Hospital São Lucas, Aracaju, SE, Brasil. Apresentado ao $20^{\circ}$ Congresso Nacional de Cirurgia Cardiaca. Maceió, AL, 2 e 3 de abril, 1993.

Laureado com o "Prêmio Nacional de Cirurgia Cardíaca - 1993".

* Do Hospital de Clínicas Dr. Augusto Leite.

Endereço para separatas: José Teles de Mendonça. Rua Francisco Porto, 1882 ap. 1001. CEP 49020-120 Aracaju, SE, Brasil. 
MENDONÇA, J.T.; CARVALHO, M.R.; COSTA, R.K.; FRANCO FILHO, E.; COSTA, G.B. - Aorto-subclavioplastia com preservação do fluxo arterial para o membro superior esquerdo. Rev. Bras. Cir. Cardiovasc., 8(2):63-82, 1993.

Acomete mais freqüentemente o sexo masculino, numa proporção de 2 a $4: 175,116$.

Embora a doença seja conhecida desde 1760 ${ }^{18}$, a primeira proposição cirúrgica só apareceu em 1944 , quando BLALOCK \& PARK ${ }^{9}$ publicaram seus experimentos para correção cirúrgica da coarctação da aorta. Propuseram seccionar a artéria subclávia esquerda e anastomosá-la distalmente na aorta descendente, após a zona coarctada. Os próprios autores admitiram que a técnica nāo promovia uma completa correção da doença.

Em 1945, trabalhando separadamente, CRAFOORD \& NYLIN ${ }^{22}$, na Suécia, e GROSS \& HUFNAGEL ${ }^{41}$, em Boston-EUA, propuseram a ressecção da zona coarctada seguida de anastomose términoterminal dos cotos aórticos, prontamente adotada por diversos centros no mundo.

A ressecção com anastomose término-terminal teve o uso comprometido nos casos em que havia longos segmentos coarctados, e que era impossível aproximar, sem tensão, os dois cotos aórticos. Além disso, a sutura circunferecial parecia ser responsável pelo aparecimento de reestenose na evolução tardia dos pacientes operados.

A partir de 1948 , GROSS et alii ${ }^{42}$ passaram a utilizar homoenxertos arteriais na reconstrução da aorta, em casos onde a coarctação era muito extensa. Posteriormente, o desenvolvimento dos enxertos plásticos tubulares veio substituir, com grandes vantagens, os homoenxertos conservados 82 .

Em 1957, VOSSCHULTE 127 introduziu novo método para tratamento da coarctação da aorta. A técnica é chamada de "istmoplastia" ${ }^{128}$ e pode ser realizada de duas maneiras distintas:

a) istmoplastia direta, indicada em curtos segmentos coarctados, consiste na incisão longitudinal, da aorta, sobre o segmento coarctado e sutura transversa das bordas;

b) istmoplastia indireta, indicada nos longos segmentos estreitados, consiste em se fazer uma incisão longitudinal sobre toda a região estreitada e, a seguir, a interposição de um enxerto losangular de material biológico ou sintético.

A impossibilidade da utilização do método direto em todos os casos e a utilização de material estranho ao organismo, no indireto, pareciam óbices à plena aceitação deste novo conceito terapêutico.

Em 1966, WALDHAUSEN \& NARHWOLD 130 introduziram o que parecia ser uma técnica irretocável para correção cirúrgica da coarctação da aorta. A técnica consiste basicamente na utilização da artéria subclávia esquerda, como enxerto vivo para ampliação do segmento estenosado, que é dissecada e ligada junto à emergência da artéria vertebral. A seguir, é aberta longitudinalmente, ampliando-se a incisão até a aorta descendente, ultrapassando-se o segmento coarctado. A artéria subclavia aberta é rebatida sobre a aorta, em forma de telhado, e suturada às suas bordas, ampliando-se largamente toda a zona coarctada.

A técnica de Waldhausen e Nahrwold havia suprimido os inconvenientes das demais; evitando a sutura circunferencial, podia ser indicada em longos segmentos estenosados e não utilizava nenhum corpo estranho pois o enxerto para alargar a aorta, era a própria subclávia, um tecido arterial vivo e, portanto, com potencial de crescimento. No entanto, a realização da técnica como foi descrita necessitava da transecção da artéria subclávia, interrompendo o suprimento sanüíneo para o membro superior esquerdo e suas conseqüências.

Em fevereiro de 1984, operando uma coarctação da aorta em criança de 6 anos de idade, encontramos um arranjo anatômico que nos pareceu favorável à realização de uma nova técnica, a qual denominamos aorto-subclavioplastia com preservação do fluxo arterial para o membro superior esquerdo 75,76 .

A técnica, como será descrita, tem todas as vantagens da aorto-subclavioplastia apresentada por WALDHAUSEN \& NAHRWOLD ${ }^{130}$ sem o seu grande inconveniente que é a secção da artéria subclávia e conseqüente interrupção do fluxo para o membro superior esquerdo.

O objetivo do presente trabalho é apresentar os detalhes técnicos que caracterizam este procedimento cirúrgico em seu estágio atual e a experiência clínica.

\section{CASUÍSTICA E MÉTODOS}

No período compreendido entre janeiro de 1984 e março de 1992, 10 pacientes portadores de coarctação da aorta foram operados na Unidade Cardiotorácica da Fundação de Beneficência, Hospital de Cirurgia - Sergipe, utilizando-se a aortosubclavioplastia com preservação do fluxo arterial para o membro superior esquerdo. Oito eram do sexo masculino e 2 do sexo feminino. A idade variou de 2 meses a 25 anos (média 6,3 anos) e o peso de $3,1 \mathrm{~kg}$ a $60 \mathrm{~kg}$ (média $21,2 \mathrm{~kg}$ ). Todos os pacientes eram sintomáticos no momento da indicação cirúrgica e os sintomas relatados foram: dispnéia em 8 pacientes; déficit pôndero-estatural em 4; crises de broncoespasmos em 3; fraqueza nos membros inferiores em 2; caimbras em membros inferiores em 1; claudicação intermitente em 1; cefaléia em 1; tonturas em 1; precordialgia em 1; cianose aos 
MENDONÇA, J.T.; CARVALHO, M.R.; COSTA, R.K.; FRANCO FILHO, E.; COSTA, G.B. - Aorto-subclavioplastia com preservação do fluxo arterial para o membro superior esquerdo. Rev. Bras. Cir. Cardiovasc., 8(2):63-82, 1993.

esforços em 1 e sudorese com irritabilidade em 1. Três pacientes apresentavam insuficiência cardíaca refratária ao tratamento clínico. Em relação aos sinais, todos apresentavam alteração na intensidade de pulso nos membros inferiores (em 9 os pulsos eram ausentes e em 1 o pulso era diminuído); 9 pacientes eram hipertensos, 4 pacientes apresentavam nítida desproporção entre as cinturas escapular e pélvica; em 3 havia hiperfonese de bulhas; em 2, hiperpulsatilidade da aorta ao nível da fúrcula esternal e 1 paciente apresentava sopro diastólico no foco aórtico.

\section{Avaliação Pré-Operatória}

O diagnóstico da coarctação da aorta foi estabelecido com base nos dados clínicos auxiliados por exames laboratoriais não invasivos, como radiografia de tórax, eletrocardiograma e ecodopplercardiograma.

Radiologicamente, o achado mais comum foi o aumento da área cardiaca, encontrado em todos os pacientes: o ventrículo esquerdo encontrava-se aumentado em 8 pacientes, o ventrículo direito em 2 e o átrio esquerdo em 2. A corrosão dos arcos costais foi vista apenas em 4 pacientes.

Ao eletrocardiograma, todos apresentavam ritmo sinusal com sinais de sobrecarga de câmaras cardíacas: sobrecarga do ventrículo esquerdo em 4, ventrículo direito em 2 , biventricular em 2 e sobrecarga de átrio e ventrículo esquerdos em 2 .

O ecodopplercardiograma só foi introduzido em nosso Serviço a partir de 1991 e foi muito útil não só na confirmação da coarctação da aorta, como também na avaliação de defeitos intracardíacos.

Todos os pacientes foram submetidos a estudo hemodinâmico invasivo para, através da aortografia, se determinarem com precisão os detalhes morfológicos de importância na programação cirúrgica. Dessa forma, as coarctações foram classificadas quanto à posição do canal arterial (KEITH et alii, em $1978{ }^{58}$ ) em: a) pré-ductal; b) justaductal, c) pós-ductal. $E$, quanto ao tipo anatômico (PELLEGRINO et alii, $1985{ }^{90}$ ), coarctações: a) em diafragma; b) em cinta; c) em estreitamento istmico.

A aortografia serviu, ainda, para determinar o desenvolvimento do istmo, bem como o arranjo anatômico da aorta e seus ramos. O estudo hemodinâmico completo foi realizado para excluir ou elucidar outras lesões cardiovasculares associadas.

Nos pacientes estudados, o tipo morfológico, em relação ao canal arterial, foi preponderantemente justaductal, encontrado em 7 pacientes; o tipo pré-ductal foi observado em 2 e pós-ductal em 1 paciente. E quanto ao tipo anatômico, 5 pacientes apresentaram coarctação em diafragma; 2 apresentaram estreitamento istmico; 2 , estreitamento ístmico mais diafragma e 1 paciente apresentava coarctação em forma de cinta.

Nove pacientes apresentavam algum tipo de defeito associado. A persistência do canal arterial (PCA) foi encontrada em 7; comunicação interventricular (CIV) em 3; hipodesenvolvimento do istmo em 3; hipoplasia do arco aórtico em 2; insuficiência aórtica em 1. Um paciente apresentava hipertensão pulmonar severa; 1 , fibroelastose do ventrículo esquerdo e outro era portador de deformidade da parede torácica - pectus carinatum.

\section{Técnica Operatória}

\section{Abordagem cirúrgica}

O acesso à cavidade torácica é feito através de uma toracotomia póstero-lateral esquerda pelo quarto espaço intercostal. O pulmão é envolvido em compressas úmidas e tracionado para adiante. A pleura mediatinal é aberta verticalmente sobre a aorta descedente e artéria subclávia esquerda, após ligadura e secção da veia intercostal superior. A borda da pleura é preparada com fios de algodão e retraída medialmente expondo, com clareza, os nervos vago e recorrente laríngeo. A aorta e a artéria subclávia são amplamente dissecadas e os ramos intercostais adjacentes à zona da coarctação são individualmente controlados com pinças tipo "bulldog" ou com fios em passagem dupla. A artéria subclávia é extensamente mobilizada, de modo a expor grande parte de seus primeiros ramos (Figura 1). 0

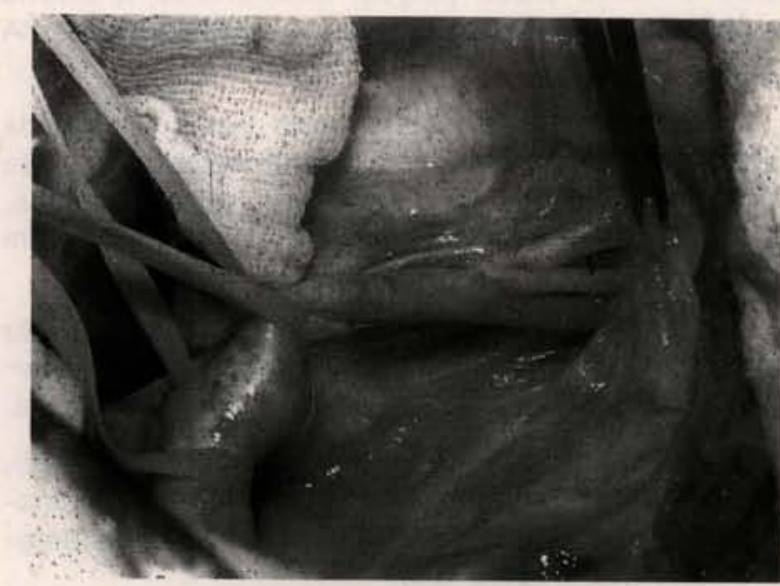

Fig. 1 - Dissecção da aorta e artéria subclávia: a foto evidencia a exposição dos principais ramos da artéria subclávia amplamente dissecados para permitir, sem tensão, maior mobilização deste vaso em direçāo à aorta descendente. 


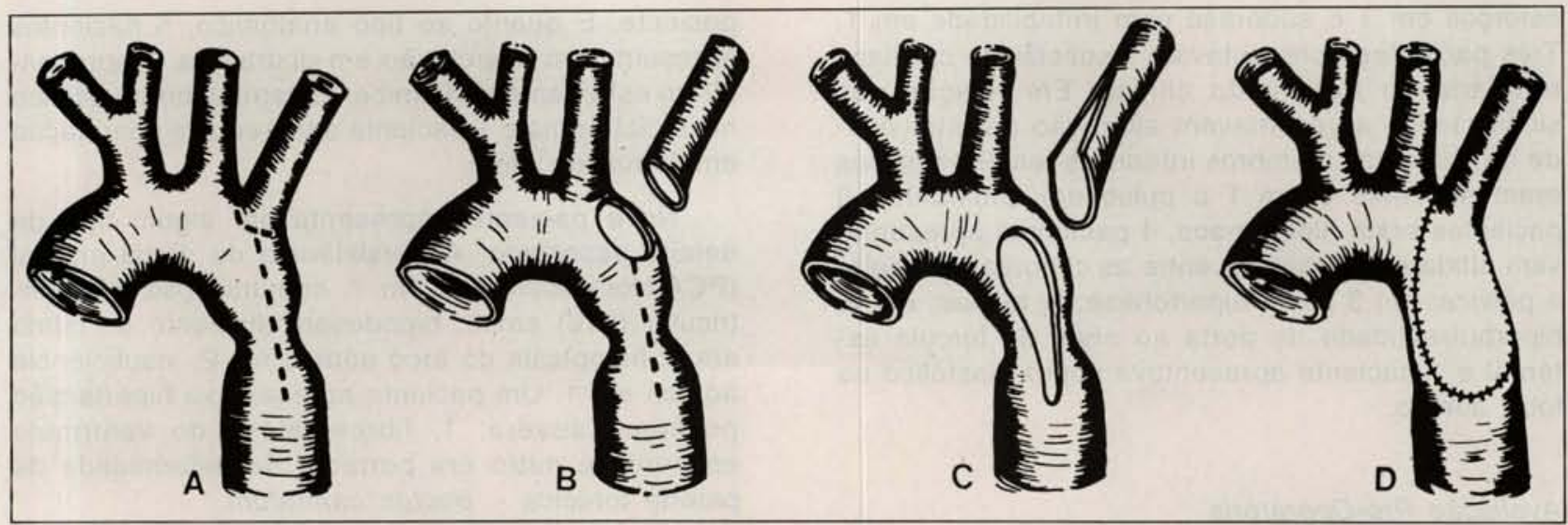

Fig. 2 - Aorto-subclavioplastia com preservação do fluxo arterial: técnica-padrão

Representação Esquemática

A: as linhas tracejadas indicam o local onde devem ser realizadas as incisōes;

B: artéria subclávia desinserida da aorta;

C: abertura da aorta e da artéria subclávia;

D: artéria subclávia já reimplantada, corrigindo a coarctação e preservando a continuidade para o braço esquerdo.

canal arterial, depois de dissecado, é ligado e seccionado. A seguir, são colocadas duas pinças vasculares na aorta: uma proximal, após a emergência da carótida esquerda, e uma distal, abaixo da zona estreitada, disposta obliquamente, de modo a deixar livre bastante parede da aorta em sua face anterior. Uma terceira pinça vascular é colocada na artéria subclávia, próximo à emergência de seus ramos principais. São utilizadas pinças vasculares anguladas ${ }^{59}$, tipo DeBakey, de tamanhos variados.

\section{Correção da coarctação aorto-subclavioplastia: técnica-padrão}

Com a aorta e a artéria subclávia devidamente pinçadas e reparados os vasos intercostais da região excluída pelas pinças, secciona-se a artéria subclávia rente à sua origem na aorta (Figuras $2 \mathrm{~A}$ e 2B).

Do orifício deixado na aorta pela retirada da artéria subclávia, inicia-se a parede anterior da aorta no sentido caudal, até ultrapassar, com segurança, todo o segmento coarctado, geralmente 12 a $15 \mathrm{~mm}$ (Figura 2C).

O diafragma fibroso, quando houver, deve ser ressecado cuidadosamente, de modo a não comprometer a resistência da parede posterior da aorta.

A artéria subclávia é aberta longitudinalmente, em sua face posterior, numa extensão aproximadamente a mesma da incisão feita na parede anterior da aorta (Figura 2C).

A artéria subclávia aberta é, então, tracionada para baixo e suturada (sutura contínua) às bordas da incisão aórtica com fio monofilamentar de
Polipropileno 5-0 ou 6-0 (Figura 2D). Antes da conclusão da sutura anterior, as pinças säo lenta e gradualmente removidas e o ar, expurgado através
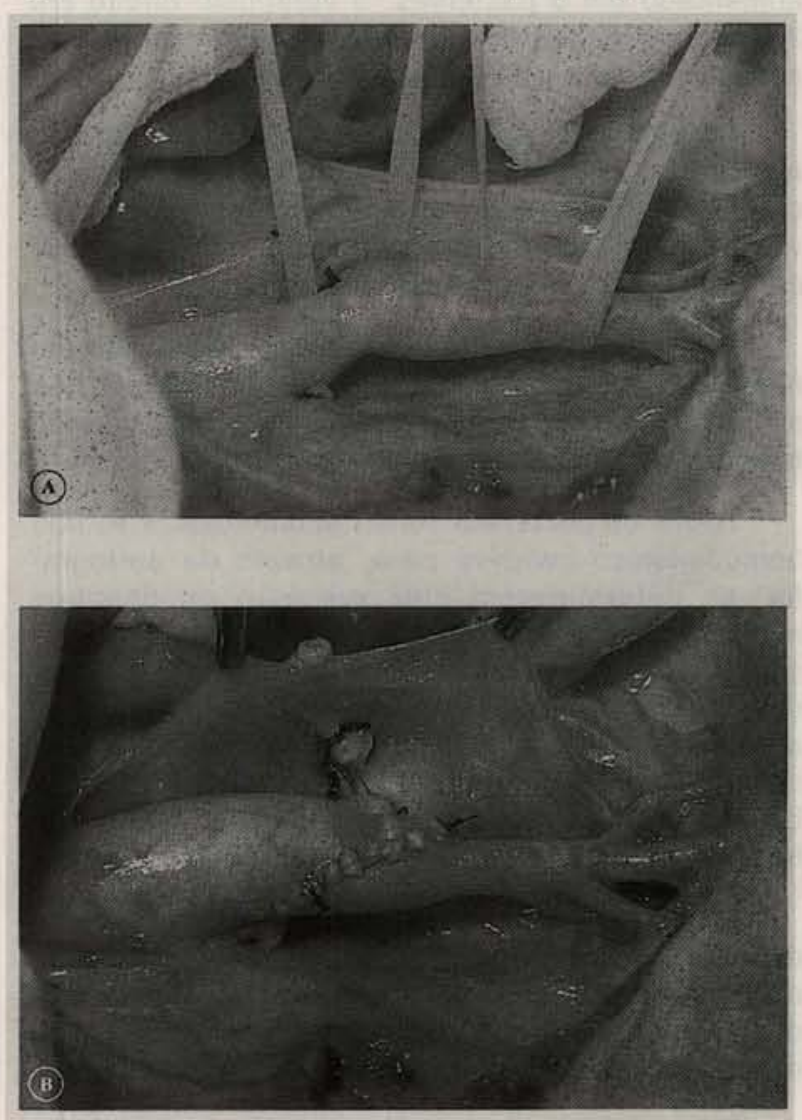

Fig. 3 - Aorto-subclavioplastia com preservação do fluxo arterial: técnica padrão aspectos cirúrgicos (paciente de $n^{\circ} 4$ ) A: antes da correção: B: cirurgia terminada. 


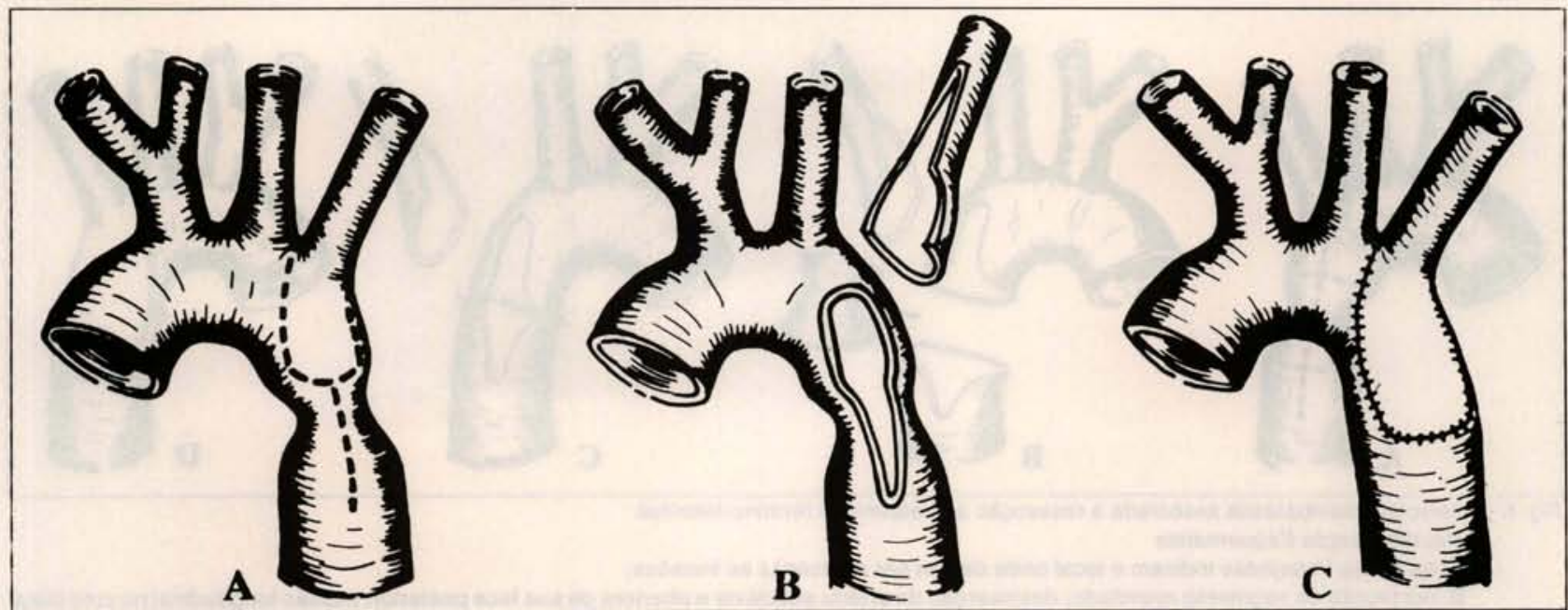

Fig. 4 - Aorto-subclavioplastia com segmento de aorta

Representação Esquemática

A: as linhas tracejadas indicam o local onde devem ser realizadas as incisões;

B: artéria subclávia desinserida conjuntamente com segemento da parede anterior da aorta: a figura mostra a artéria subclávia e a aorta devidamente abertas:

C: artéria subclávia já reimplantada conjuntamente com o segmento, corrigindo a coarctação e preservando a continuidade arterial para o braço esquerdo.

da linha de sutura. Conclui-se a sutura e revisa-se cuidadosamente a hemostasia.

A Figura 3 mostra uma seqüência cirúrgica na qual foi utilizado esse procedimento.

\section{Aorto-subclavioplastia com segmento de aorta}

Consiste na retirada de um fragmento da aorta em conjunto com a artéria subclávia (Figura 4). No mais, o procedimento é extamente igual ao anterior.

A manobra deve ser utilizada naqueles pacientes em que a distância entre o estreitamento e a emergência da artéria subclávia seja grande e o istmo bem desenvolvido. Esse recurso técnico permite uma excelente ampliação da zona estreitada com pequeno deslocamento da artéria subclávia (Figura 5).

\section{Aorto-subclavioplastia associada a ressecção e anastomose término-terminal}

O procedimento tem iniçio com a coartectomia e conseqüente retirada do tecido remanescente do canal arterial (Figuras 6A e 6B).

A seguir, procede-se à desinserção da artéria subclávia e amplia-se inferiormente o orifício criado até a borda livre da aorta. No coto distal da aorta, face anterior, pratica-se uma pequena incisão longitudinal (Figura 6B).
Inicia-se a reconstrução, com anastomose término-terminal, unindo apenas a parede posterior (Figura 6C). Utiliza-se sutura contínua com fio monofilamentar de Polipropileno 5-0 ou 6-0.

Posteriormente, e de modo similar à técnicapadrão, reimplanta-se a artéria subclávia devidamente aberta, interposta entre as bordas da fenda aórtica, ampliando-se sobremaneira essa região, sem deixar qualquer sutura circunferencial (Figura 6D).

A Figura 7 mostra uma seqüência cirúrgica na qual foi utilizado esse procedimento. Nos pacientes de números 1, 3 e 4 utilizou-se a aorto-subclavioplastia simples (técnica-padrão). Nos pacientes de números 2 e 5 foi utilizada a aorto-sub-

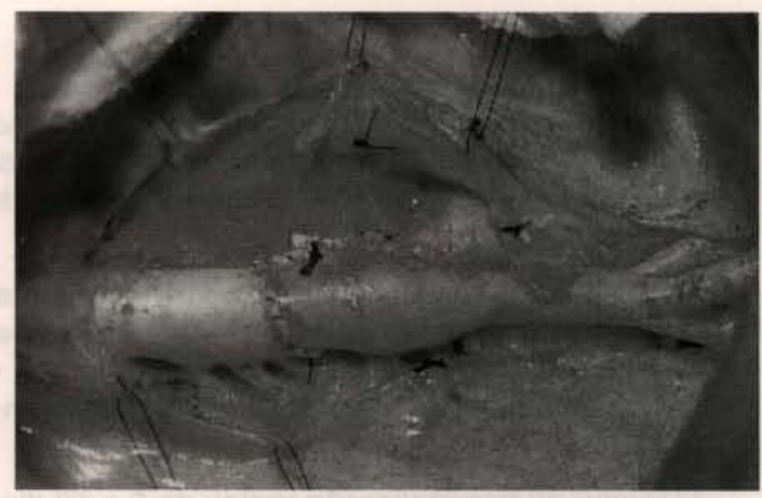

Fig. 5 - Aorto-subclavioplastia com segmento da aorta: foto cirúrgica - aspecto final (cortesia Dr. Milton Meier). 


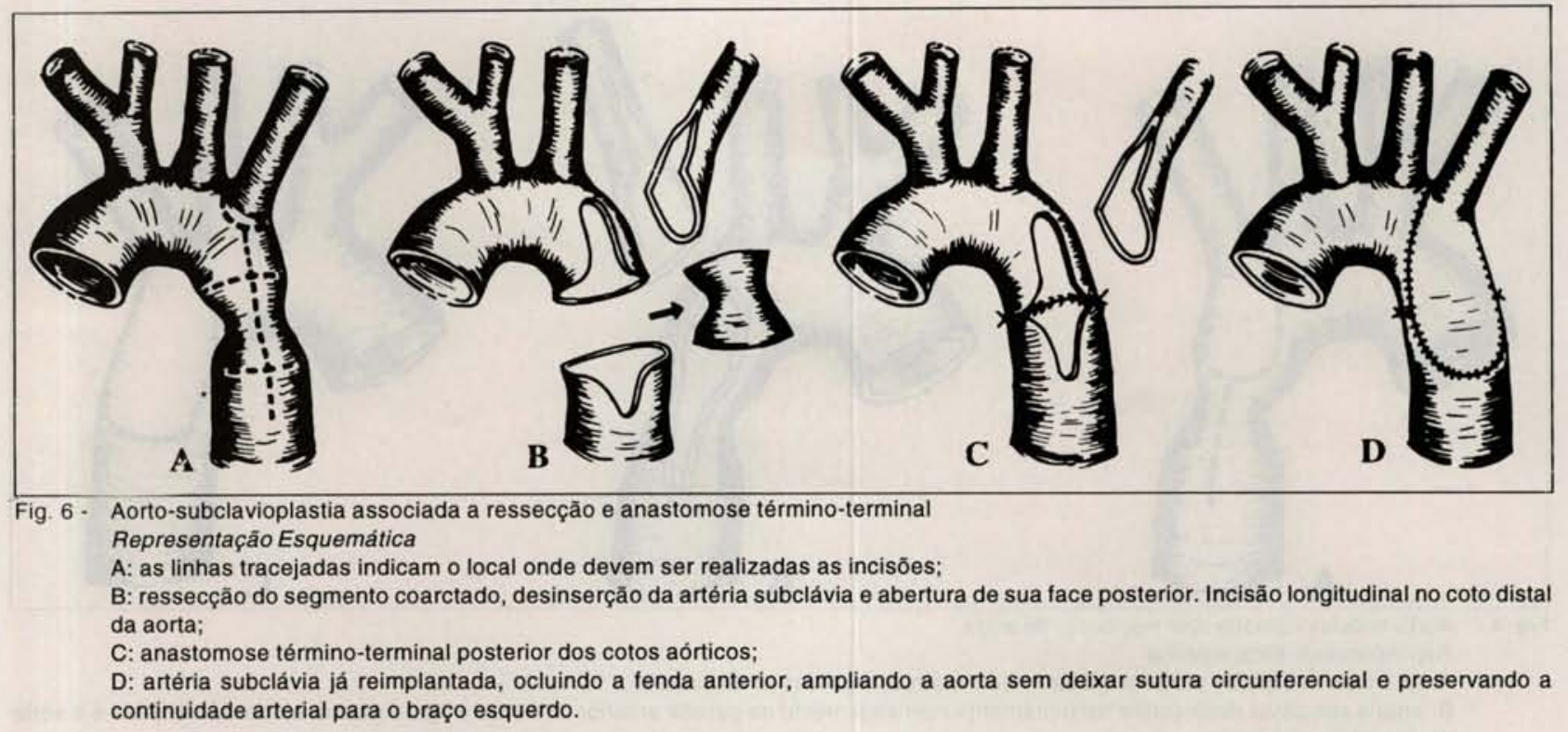

clavioplastia com segmento de aorta e nos pacientes de números $6,7,8,9$ e 10 empregou-se a aorto-subclavioplastia associada com ressecção e anastomose término-terminal. $O$ único procedimento cirúrgico associado foi a ligadura e secção do canal arterial.

Após revisão da hemostasia, a pleura mediastinal é aproximada com sutura contínua de Polipropileno 5-0. A cavidade pleural é drenada (dreno plástico tubular) e a parede torácica, fechada em quatro planos. As costelas são aproximadas com pontos pericostais de categute cromado 0 ou 00. O plano muscular é suturado com pontos separados de algodão ou "mononylon" 00 . O subcutâneo, com categute simples 00 ou 000 e, finalmente, uma sutura intradérmica com poliglactina (Vicril) 0000. Cobre-se a ferida com curativo seco.

\section{RESULTADOS}

Nove pacientes suportaram satisfatoriamente a intervenção. Houve apenas um óbito operatório. Tratava-se do paciente de número 10 , portador de coarctação pré-ductal, PCA e fibroelastose do ventrículo esquerdo.

Dois pacientes (de números 5 e 6) apresentaram hipertensão paradoxal no pós-operatório imediato, cedendo com o uso de betabloqueador e inibidores da enzima conversora da angiotensina (ECA).

O tempo de hospitalização variou de 5 a 10 dias (média de 7 dias) e todos os 9 pacientes que sobreviveram foram seguidos em regime ambulatorial.
O tempo de seguimento mínimo de 12 meses e o máximo de 8 anos (média de 3,9 anos). Todos os pacientes mantiveram-se assintomáticos. Apresentavam, no último controle, pulsos normais nos membros inferiores e membro superior esquerdo e, com a pressão arterial, em repouso, dentro dos limites da normalidade. Um paciente (de múmero 2), o mais velho da série, evolui com pressão arterial normal, porém em uso de diuréticos tiazídicos $e$ inibidores da ECA.

O estudo angiográfico pós-operatório foi realizado entre o segundo dia e o sexto ano de seguimento (média de 19 meses). Em 8 pacientes foi observada uma excelente ampliação da aorta com preservação do fluxo para o membro superior esquerdo, através da artéria subclávia reimplantada. Em apenas 1 paciente, de número 7 , observou-se pequeno estreitamento ao nível da zona operada, que produzia gradiente de $20 \mathrm{mmHg}$. A paciente encontra-se assintomática, com pressão e pulsos normais.

As Figuras 8, 9 e 10 mostram angiogramas pré e pós-operatórios de pacientes operados com a técnica-padrão e suas variantes.

\section{COMENTÁRIOS}

Passado quase meio século da primeira operação para correção da coarctação da aorta, dois pontos fundamentais ainda não foram devidamente esclarecidos: Quando? e Como? operar.

As dúvidas seriam facilmente dirimidas, não fosse a coarctação da aorta uma doença com largo 

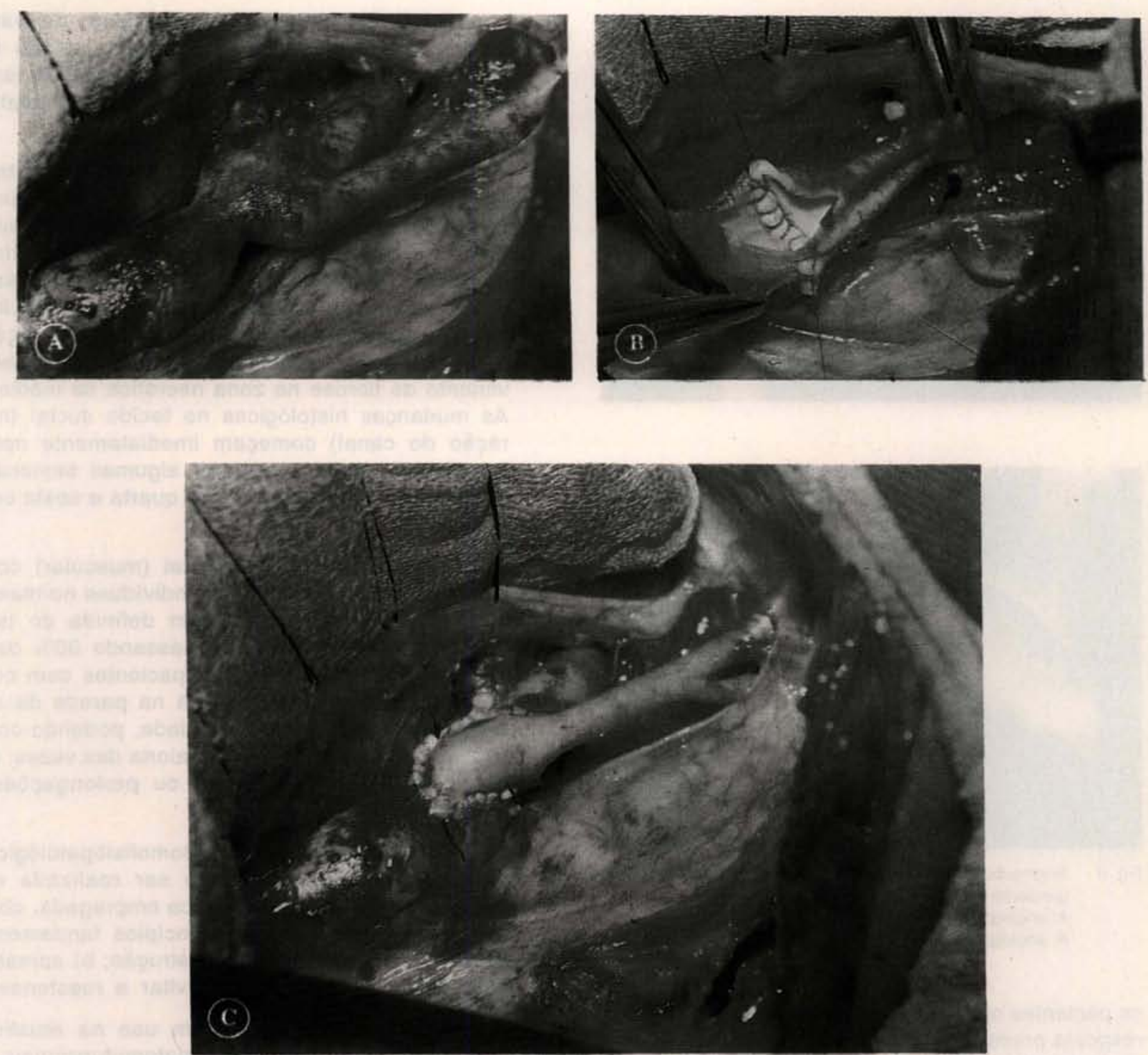

Fig. 7- Aorto-subclavioplastia associada a ressecção e anastomose término-terminal: aspectos cirúrgicos (paciente de $n^{2} 6$ ) A: aspecto inicial após dissecçāo da aorta e artéria subclávia;

B: tempo intermediário, após ressecção do segmento coarctado e anastomose término-terminal posterior dos cotos aórticos;

C: cirurgia concluída com a artéria subclávia reimplantada ampliando a aorta e preservando a continuidade arterial para o braço esquerdo.

espectro de apresentação e com características anatomopatológicas especiais que mudam, inclusive, no decorrer da vida, tornando, algumas vezes, difícil a escolha da técnica mais apropriada, bem como a determinação do momento exato para a indicação cirúrgica $2,30,74,77,119$.

A coartação da aorta representa um obstáculo ao livre fluxo de sangue através da aorta, produzindo um regime hipertensivo no território proximal e hipotensivo no território distal. Tanto a hipertensão proximal como a hipotensão distal são responsáveis por alterações importantes no sistema cardiovascular, as quais podem perdurar, mesmo após a correção cirúrgica. A hipertensão, além de produzir sobrecarga do ventrículo esquerdo e posterior descompensação ${ }^{117}$, produz mudanças estruturais importantes nas paredes dos vasos 103, 107, 110 e altera significativamente a função dos baro-receptores aórticos e caróticos 106 .

O regime de baixo fluxo no território distal produz alterações no sistema renina-angiotensina 88,94 .

Todos esses fatores são responsáveis pela manutenção da pressão arterial elevada após a correção cirúrgica ou, quando menos, fazem com que 
MENDONÇA, J.T.; CARVALHO, M.R.; COSTA, R.K.; FRANCO FILHO, E.; COSTA, G.B. - Aorto-subclavioplastia com preservação do fluxo arterial para o membro superior esquerdo. Rev. Bras. Cir. Cardiovasc., 8(2):63-82, 1993.
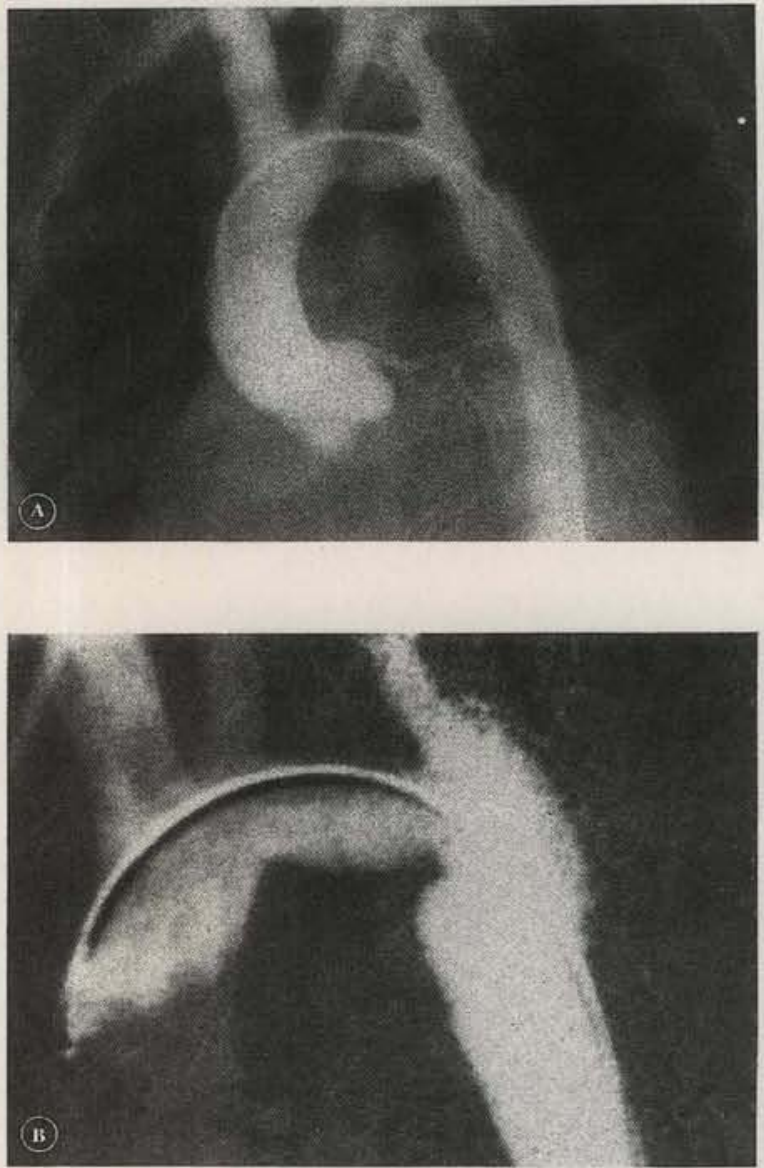

Fig. 8- Aorto-subclavioplastia-técnica-padrão: aspectos angiográficos (paciente de $n^{\circ} 4$ )

A: angiografia pré-operatória;

B: angiografia pós-operatória, 84 meses após a correção.

os pacientes operados tardiamente apresentem uma resposta pressórica anormal ao exercício $1,39,56,57$, $69,84,108,111,129$.

O momento exato a partir do qual estas alterações tornam-se irreversíveis não foi, ainda, claramente definido, embora haja entre os autores o consenso de que a indicação cirúrgica deva ser feita eletivamente o mais precoce possível $84,91,110$.

Outros fatores, como a polimórfica apresentação da doença e a participação do tecido ductal na parede da aorta são considerados complicadores, tanto na escolha do procedimento, como na determinação do momento operatório.

A coarctação da aorta é uma entidade anatomopatológica complexa que, do ponto de vista antômico, pode assumir três formas básicas de apresentação, isoladas ou associadas. São elas: a) em diafragma; b) introflexão da parede da aorta formando uma cinta; c) estreitamento ístmico 90 .
Histologicamente, o envolvimento da parede aórtica pelo tecido ductal é o fator isolado mais importante na determinação dos resultados tardios na cirurgia da coarctação da aorta em neonatos e lactentes $5,19,27,30,70,104,136$.

O tecido ductal difere da parede da aorta por ser essencialmente muscular, com uma camada elástica fragmentada e uma fina camada intima ${ }^{91}$. Esse tecido especial segue uma seqüência de mudanças histológicas após o nascimento, a saber: a) espessamento da camada média, proliferação da íntima e contração dos vasos; b) depósitos mucóides e degeneração isquêmica da média; c) desenvolvimento de fibrose na zona necrótica da média ${ }^{114}$. As mudanças histológicas no tecido ductal (maturação do canal) começam imediatamente após o nascimento, podem perdurar algumas semanas $e$, usualmente, terminam entre a quarta e sexta semana 100,101 .

A junção do tecido ductal (muscular) com o tecido da aorta (elástico) em indivíduos normais dáse com uma penetração bem definida do tecido ductal na aorta, nunca ultrapassando $30 \%$ da circunferência desse vaso. Em pacientes com coarctação, o tecido ductal penetra na parede da aorta em graus variados de intensidade, podendo ocupar toda sua circunferência. Na maioria das vezes, esse tecido ductal emite línguas ou prolongações no sentido distal $30,53,102$.

Diante da realidade anatomofisiopatológica, a cirurgia de coarctação deve ser realizada eletivamente na infância e a técnica empregada, obedecer rigorosamente a dois princípios fundamentais: a) aliviar completamente a obstrução; b) apresentar potencial crescimento para evitar a reestenose.

As inúmeras técnicas em uso na atualidade podem, por características anatomofuncionais, ser

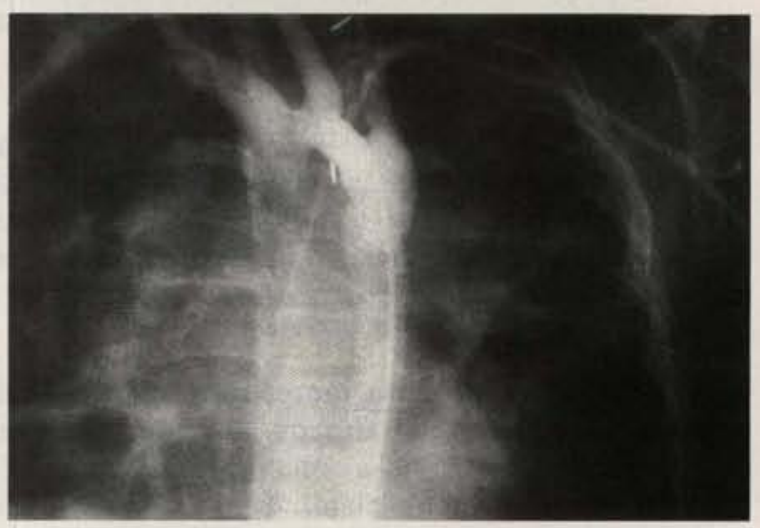

Fig. 9 - Aorto-subclavioplastia com segmento da aorta: aspecto angiográfico pós-operatório, 12 meses após a correção (cortesia Dr. Milton Meier) 
MENDONÇA, J.T.; CARVALHO, M.R.; COSTA, R.K.; FRANCO FILHO, E.; COSTA, G.B. - Aorto-subclavioplastia com preservação do fluxo arterial para o membro superior esquerdo. Rev. Bras. Cir. Cardiovasc., 8(2):63-82, 1993.
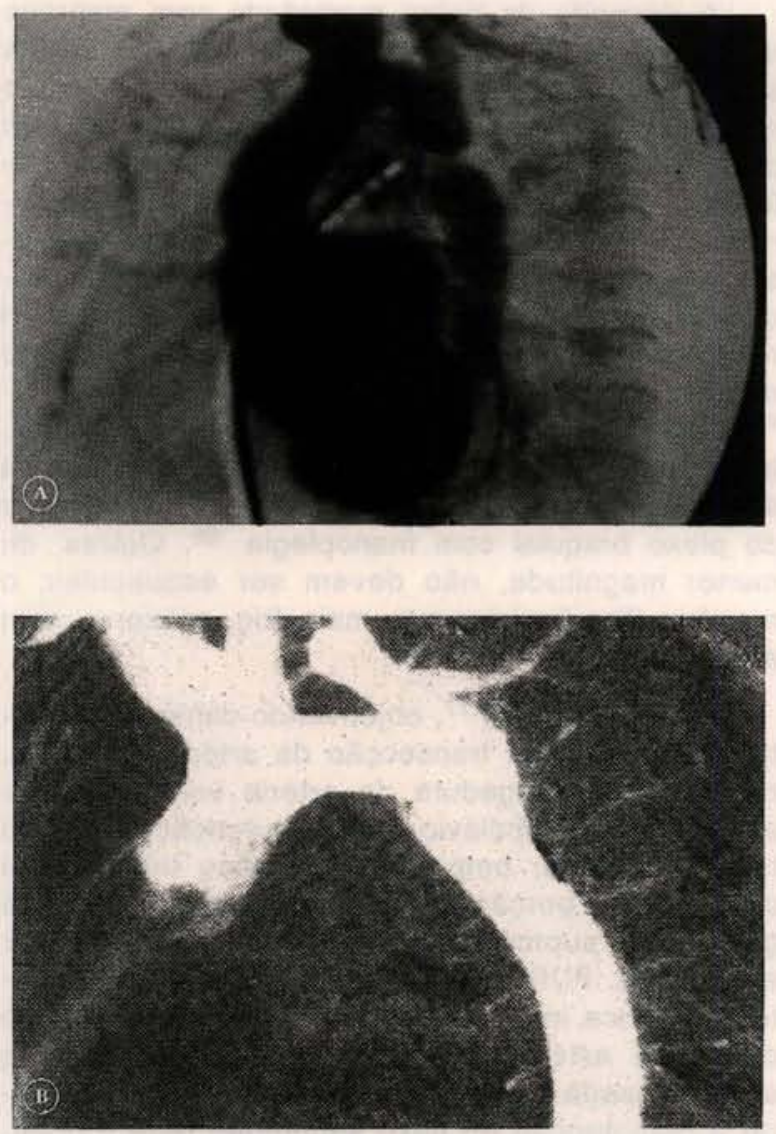

Fig. 10 - Aorto-subclavioplastia associada a ressecção e anastomose término-terminal: aspectos angiográficos (paciente de $n^{2} 8$ ) A: angiografia pré-operatória;

B: angiografia pós-operatória, 12 meses após a correção.

resumidas em três grupos: a) ressecção e anastomose término-terminal; b) istmoplastia; c) aortosubclavioplastia. Todas com vantagens e desvantagens, críticos e apologistas. A ressecção e anastomose término-terminal 22,42 tem, como grande vantagem sobre as demais, a possibilidade de, ressecando o fragmento estreitado, eliminar o remanescente do tecido ductal responsável pela, reestenose durante o processo de maturação, quando a cirurgia é realizada nos primeiros meses de vida $19,62,77,121,136$. Entretanto, esta técnica deixa, obrigatoriamente, uma sutura circunferencial na aorta, que tem sido responsabilizada, por muitos, pela falta de crescimento no sítio da anastomose e conseqüentemente, elevados índices de recoarctação $21,41,59,111,115,133$. Outros autores têm obtido índices relativamente baixos de recoarctação com este procedimento, mesmo quando operam crianças no primeiro ano de vida $31,56,62,101$. Atribuem o aparecimento da reestenose a outras causas, tais como lesão da aorta por pinças vasculares 31 ; ressecção incompleta; restos de tecido ductal 11,99 e falhas técnicas, tais como a realização de suturas fortemente tracionadas que levam a um esgarçamento da íntima e conseqüente proliferação do tecido fibroso no local da sutura 62 . KORFER et alii 62 chegam a afirmar que, com uma larga dissecção, remoção de todo o tecido doente e realização da anastomose com pontos separados, sem tração, a aorta irá crescer e a recoarctação será rara.

Para eliminar o principal inconveniente deste procedimento, algumas modificações técnicas foram introduzidas, com o objetivo comum de eliminar a sutura circunferencial e ampliar ao máximo a boca anastomótica, sendo, por isso, chamadas genericamente de: ressecção com anastomose términoterminal estendida.

O procedimento cirúrgico consiste, basicamente, na ressecção, a mais ampla possível, do segmento estreitado e, para ampliar a área de sutura, estende-se a incisão no coto proximal em direção à artéria subclávia ${ }^{45}$, ou em direção à parede inferior do arco aórtico, quando este for hipoplásico $3,29,66,135$. No coto distal a extensão da incisão é feita na face externa ou interna da aorta, a depender da incisão feita no coto proximal, de modo a compatibilizar o tamanho das bocas anastomóticas.

Esta operação tem sido recomendada com veemência por alguns autores $64,112,120,127$ sobretudo para neoatos com hipoplasia do arco aórtico. Por outro lado, SIEWERS et alii ${ }^{112}$, só recomendam a ressecção com anastomose término-terminal estendida em pacientes com hipoplasia acentuada do arco aórtico (diâmetro do arco menor ou igual a $25 \%$ do diâmetro da aorta ascendente) uma vez que este procedimento acarreta maior morbi-mortalidade e, o que é mais importante, estes arcos hipoplásicos podem crescer após a correção convencional, como foi demonstrado por BROUWER et alii. ${ }^{13}$.

Nos casos de hipoplasia localizada na porção distal do arco aórtico, VINCENT et alii ${ }^{124}$ propõem que se faça uma ressecção com anastomose término-terminal clássica associada a uma plastia localizada do arco aórtico, utilizando-se a artéria subclávia.

Neste grupo, apesar dos avanços, permanecem como pontos críticos: a) impossibilidade de usar em longos segmento coarctados, principalmente nas crianças maiores e adultos 60 ; b) necessidade de longas dissecções com possibilidades de lesar estruturas importantes, tais como ducto linfático, nervos vago e recorrente e vasos intercostais $78 \%$; c) sutura circunferencial que, mesmo com perfeita ampliação, funciona como um anel rígido, fazendo aparecer gradientes durante o exercício 36,123 .

A istmoplastia 125,126 é um procedimento extremamente simples e tem como pontos positivos: a) 
MENDONÇA, J.T.; CARVALHO, M.R.; COSTA, R.K.; FRANCO FILHO, E.; COSTA, G.B. - Aorto-subclavioplastia com preservaçāo do fluxo arterial para o membro superior esquerdo. Rev. Bras. Cir. Cardiovasc., 8(2):63-82, 1993.

fácil de executar, exigindo um mínimo de dissecções e preservando todas as estruturas intactas 77 . $80,81,134$; b) possibilidade de correção da doença sem necessidade de usar corpo estranho, quando a istmoplastia direta puder ser utilizada: c) o tamanho do segmento coarctado, virtualmente, não tem importância, pois o enxerto (istmoplastia indireta), pode progredir em direção ao arco aórtico e/ou subclávia ${ }^{77}$; d) potencial de crescimento, pois, tanto a parede posterior da aorta como o ístmo hipoplásico, podem crescer $20,23,95,102,135$.

A impossibilidade da utilização da istmoplastia direta em todos os casos e emprego de material estranho na istmoplastia indireta são os pontos críticos deste procedimento. O uso de enxerto, quer seja biológico ou sintético, está relacionado a dois fatos negativos na evolução dos pacientes. Em primeiro lugar, o enxerto não cresce $e$, quando a operação é realizada em crianças muito pequenas, existem grandes possibilidades de reestenose ao longo do tempo 45, 51, 98 .

Um outro problema está relacionado à resistência dos materiais. O enxerto rígido apresenta uma resistência diferente daquela encontrada na parede posterior da aorta, fazendo com que seja transmitida uma tensāo adicional à parede do vaso que, sustentando a carga total da onda de pulso, sofre dilatação 7,86 . A formação de aneurismas é a principal complicação deste procedimento 12 , $47,48,96$.

Outros fatores têm sido relacionados como causa de aneurisma nesta operação; alterações estruturais na parede da aorta 12,54 e enfraquecimento da parede posterior por ressecção exagerada do tecido ductal $24,25,47$.

$\mathrm{Na}$ tentativa de otimizar esta técnica cirúrgica CAMPALANI et alii ${ }^{15}$, MOOR et alii ${ }^{80}$ propõem a utilização de um enxerto livre da artéria torácica interna. Desta forma, o emprego de um tecido vivo, além de apresentar a mesma resistência, teria o benefício adicional de poder crescer. Recentemente, CATANI et alii ${ }^{17}$, HENZE \& HALLBERG 49,50 apresentaram variantes nas quais sugerem utilizar a artéria torácica interna esquerda como enxerto pediculado, com os mesmós objetivos. O único problema relativo ao emprego dessa artéria é o seu tamanho reduzido, especialmente nas crianças menores, quando a operação deve ser eletivamente indicada.

A aorto-subclávioplastia proposta por WALDHAUSEN \& NAHRWOLD 130 descartam os dois inconvenientes das técnicas anteriores. Não utilizam sutura circunferencial e o enxerto empregado para ampliação da aorta é a própria subclávia; portanto, com real potencial de crescimento $43,83,87,92$.
A despeito da baixa morbidade com emprego desta técnica $4,8,16,91,93$, transecção da artéria subclávia com interrupção do fluxo arterial para o membro superior esquerdo não deixa de ser um fato preocupante. A artéria subclávia transectada e utilizada nas operações de derivação sistêmico-pulmonar ${ }^{10}$ e na aorto-subclavioplastia ${ }^{128}$, pode causar isquemia importante do membro superior esquerdo em menos de $1 \%$ dos pacientes ${ }^{8}$. Existem relatos de isquemia aguda, gangrena e até perda do membro superior após este procedimento $37,61,71$. 131. Outras conseqüências têm sido mencionadas, algumas delas com repercussão significativa: a síndrome do roubo subclávio $33,67,79,85$ e a injúria do plexo braquial com manoplegia 68 . Outras, de menor magnitude, não devem ser esquecidas; o membro fica ligeiramente mais frio, menor e com menos força $23,43,68,118$.

MESMER et alii ${ }^{77}$, objetivando diminuir os efeitos deletéricos da transecção da artéria subclávia, recomendam a ligadura da artéria vertebral, para evitar o roubo subclávio, e a manutenção da artéria torácica interna, bem como o tronco tireocervical conectados à porção distal da artéria subclávia, para garantir um suprimento colateral de sangue e evitar $a$ isquemia. FURNIER et alii ${ }^{34}$ propõem que a artéria torácica interna esquerda conectada à porção distal da artéria subclávia seja seccionada e anastomosada distalmente na aorta, para assegurar a manutenção do fluxo sangüíneo para o membro esquerdo.

Aorto-subclavioplastia com preservação do fluxo arterial para o membro superior esquerdo apresenta, teoricamente, todos os pontos positivos da aorto-subclavioplastia proposta por WALDHAUSEN \& NAHRWOLD ${ }^{130}$, sem o seu grande inconveniente, a transecção da artéria subclávia.

Posteriormente, com o uso clínico difundido, duas modificaçōes foram introduzidas. A primeira, proposta por MEIER et alii ${ }^{73}$, consiste na utilização de um segmento da aorta que é retirado conjuntamente com a artéria subclávia. Esta manobra permite uma excelente ampliação com um deslocamento menor da artéria subclávia e deve ser usada naqueles pacientes possuidores de um istmo bem formado.

Outra modalidade de aplicação desta técnica representa, na realidade, uma associação de dois procedimentos: a "ressecção com anastomose término-terminal" e a "aorto-subclavioplastia com preservação do fluxo arterial" 26,73. Esta associação permite, com a ressecção da área coarctada, a remoção parcial ou total do tecido ductal. A reconstrução da aorta com anastomose término-terminal, posterior, e a interposição da artéria subclávia entre os cotos aórticos, anteriormente, além de não dei- 
MENDONÇA, J.T.; CARVALHO, M.R.; COSTA, R.K.; FRANCO FILHO, E.; COSTA, G.B. - Aorto-subclavioplastia com preservação do fluxo arterial para o membro superior esquerdo. Rev. Bras. Cir. Cardiovasc., 8(2):63-82, 1993.

xar nenhuma sutura circunferencial, promove uma excelente remodelação anatômica com uma ampliação que, na maioria das vezes, ultrapassa o diâmetro normal da aorta.

A nossa experiência com a técnica original e suas modificações tem fornecido resultados imediatos e tardios animadores. De 10 pacientes operados, houve um óbito não relacionado com o procedimento cirúrgico. Tratava-se de um paciente com dois meses (paciente de número 10), portador de coarctação pré-ductal, PCA e fibroelastose do ventrículo esquerdo. Foi operado em condições críticas (insuficiência cardíaca incontrolável e acidose metabólica mantida) e, no final da operação, ainda com o tórax aberto, apresentou parada cardíaca, que não respondeu às manobras de ressuscitação. Os sobreviventes foram seguidos ambulatorialmente e, no reestudo, apenas 1 paciente apresentou leve estrangulamento com gradiente de 20 $\mathrm{mmHg}$, através da zona operada. Nos demais, nenhum gradiente foi notado e observou-se, nos angiogramas, uma perfeita configuração anatômica, com crescimento harmônico de toda a região e a artéria subclávia irrigando plenamente o membro superior esquerdo.

Outras experiências têm sido relatadas. MEIER et alii ${ }^{73}$, em trabalho multicêntrico, relatam a experiência com 28 pacientes na faixa etária variando entre 2 meses e 25 anos (média 4,9 anos). No estudo, não houve óbitos imediatos e os resultados encontrados demonstraram ter havido uma perfeita correção. Quatro pacientes foram recateterizados entre o quarto e o nono mês e, em todos, foi observada uma adequada correção, inclusive com forte evidência de crescimento da aorta no sítio da coarctação, bem como preservação do fluxo arterial através da artéria subclávia. Nos demais pacientes, medidas com Doppler mostraram fluxo normal para o braço esquerdo e ausência de gradientes através da área tratada.

Em contrapartida, LADUSANS et alii ${ }^{65}$ relatam recoarctação precoce em 4 pacientes em que utilizaram esta técnica. Os pacientes tinham idade de 17 dias, 10 dias, 3 dias e 2 anos, no momento da operação. MESMER et alii 77 , em crianças com menos de 1 ano, encontraram uma incidência de recoarctação em torno de $+3 \%$. DIETL et alii 27 não encontraram nenhuma estenose residual ou recoarctação em 18 crianças com menos de 1 ano de idade, em que utilizaram a aorto-subclavioplatia ( 3 das quais com preservação do fluxo) associada a ressecção e anastomose término-terminal em um período de observação superior a 50 meses e concluem que a técnica combinada é o melhor procedimento para neonatos.

Mais recentemente, SHARMA et alii ${ }^{109}$ relatam a experiência com aorto-subclavioplastia (técnicapadrão) que foi empregada em 7 neonatos (idade entre 3 e 30 dias). O tempo médio de seguimento foi de 2 anos, e apenas uma criança apresentou gradiente significativo ao reestudo $(30 \mathrm{mmHg})$ e que desapareceu após angioplastia com cateter balão.

A razão de resultados tão discrepantes parece estar assentada em dois grandes pilares: a) execução técnica; b) idade no momento da operação.

Em relação aos detalhes técnicos, se faz mister chamar atenção para alguns pontos fundamentais na realização da aorto-subclavioplastia com preservação do fluxo arterial. Em primeiro lugar, é necessária uma dissecção ampla da aorta (descendente e arco) bem como a porção inicial dos vasos intercostais, sem, no entanto, haver necessidade de os sacrificar. A artéria subclávia deve ser dissecada extensamente de modo a expor grande parte dos seus principais ramos, quando a artéria for tracionada para baixo.

Outro ponto de extraordinária importância é que a incisão feita distalmente na aorta deve ultrapassar com segurança toda a zona estreitada. Em geral, isto se consegue ultrapassando-se em $12 \mathrm{~mm}$ a $15 \mathrm{~mm}$ a área de maior estrangulamento.

A operação feita desta maneira permite uma ampliação adequada de todo o segmento coarctado, com enxerto vivo que pode crescer representando, aproximadamente, dois terços da circunferência no local de maior estrangulamento. Este fato garante um adequado diâmetro luminal, mesmo havendo retração fibrótica da parede posterior, por maturação do canal ou por proliferação da íntima estimulada por distúrbios remanescentes de fluxo. Uma ampla dissecção dos vasos envolvidos promove adequada mobilização, permitindo que se faça uma sutura sem tração e, conseqũentemente, mantendo íntegro o endotélio vascular. Evitam-se, a todo custo, as lacerações vasculares responsáveis pela proliferação do tecido fibroso ao longo das suturas e, em conseqüência, maior incidência de reestenose.

O segundo pilar de interferência nos resultados é a idade no momento da operação. E isto, sem dúvida, está diretamente relacionado ao maior envolvimento da aorta pelo tecido ductal. Como a maturação do tecido ductal se faz com retração fibrótica e proliferação da íntima ${ }^{101}$, é de se esperar uma maior incidência de recoarctação naqueles pacientes operados na fase em que o processo de involução do canal ainda não se completou. De fato, a literatura tem demonstrado que a incidência de recoarctação é maior quando a operação é realizada no primeiro ano de vida e, excepcionalmente, muito maior quando realizada nos primeiros três meses, independentemente da técnica utilizada 6 . $19,58,63,78,91,101,104,105,111$. 
MENDONÇA, J.T.; CARVALHO, M.R.; COSTA, R.K.; FRANCO FILHO, E.; COSTA, G.B. - Aorto-subclavioplastia com preservaçāo do fluxo arterial para o membro superior esquerdo. Rev. Bras. Cir. Cardiovasc., 8(2):63-82, 1993.

É necessário afirmar que a coarctectomia não assegura a eliminação de todo o tecido ductal, uma vez que existem prolongações desse tecido na parede da aorta, aparentemente normal, aos olhos desarmados do cirurgião, e sua permanência pode justificar o aprecimento precoce da recoarctação, em pacientes em que a técnica operatória parecia perfeita 90, 101.

Especialmente em relação à aorto-subclavioplastia, com ou sem preservação do fluxo arterial, dois fatos existem sugerindo a participação do tecido ductal nesta porção da aorta, como agente causador de reestenose: a) as recoarctaçōes, quando a operação é realizada no período neonatal, aparecem precocemente nas primeiras 4 a 6 semanas, portanto, dentro do período de maturação do tecido ductal; b) via de regra a estenose é distal, fora da área coberta pela artéria subclávia 28,101 , 109. A recoarctação seria, então, causada pela retração do tecido ductal na parede da aorta além da zona ampliada com a artéria subclávia. Os casos apresentados por LADUSANS et alii 65 e a única reestenose de nossa série dão a noção exata deste fenômeno.

Hoje, está bastante claro que o cirurgião deve ter em mente alguns artifícios técnicos para eliminar ou diminir os efeitos maléficos desse tecido especial, sobretudo, quando o paciente a ser operado tiver menos de 1 ano de idade e, principalmente, menos de 3 meses.

Utilizando-se a aorto-subclavioplastia com preservação do fluxo arterial (técnica-padrão), isto é conseguido com uma longa incisão que ultrapasse folgadamente todo o segmento estreitado. A artéria subclávia, depois de amplamente mobilizada, deve ser reimplantada o mais baixo possívet.

Quando se emprega a aorto-subclavioplastia com preservação do fluxo arterial associada a ressecção e anastomose término-terminal, parte do objetivo já é conseguida com a ressecção da área coarctada e, para evitar remanescentes do tecido ductal contornando o coto distal da aorta, realizase uma incisão longitudinal, de modo que a artéria subclávia, quando reimplantada, ultrapasse o local da anastomose posterior.

Finalmente, a escolha do procedimento para correção cirúrgica da coarctação da aorta deixa de ser só um ato preferencial de uma equipe médica para tornara-se, também, conseqüência da análise individual de vários fatores, destacando-se, entre eles: a idade no momento da operação e a anatomia estrutural dos vasos envolvidos.

Cabe, portanto, às equipes cirúrgicas familiarizarem-se com as diversas técnicas e, ainda assim, estarem preparadas para, de quando em vez, utilizarem procedimentos poucos ortodoxos. Desta maneira, para os casos complicados com importantes alteraçōes estruturais da aorta, em operações simultâneas ou reoperações, podem-se utilizar procedimentos de derivação com tubos plásticos ou biológicos, unindo o território proximal ao território distal da aorta, deixando intacta a região coarctada $14,39,40,82,95,115,133$.

Ao nosso ver, a aorto-subclavioplastia com preservação do fluxo arterial (técnica-padrão ou associada) pode ser empregada na grande maioria das coarctações tratadas na idade pediátrica; assegura uma correção anatomicamente adequada e permite um crescimento normal da aorta, mesmo quando empregada em lactentes e neonatos $28,73,92,97,109$. 126, 138 .

\section{CONCLUSÕES}

1) A aorto-subclavioplastia com preservação do fluxo arterial - (técnica-padrão ou com as modificações introduzidas) é um procedimento técnico viável e facilmente reproduzível.

2) Tecnicamente aproxima-se do procedimento ideal para correção da coarctação da aorta, pelas seguintes razões:

a) Promove completo alívio da obstrução

b) Não utiliza nenhum material estranho ao organismo

c) Não sacrifica qualquer parte da árvore arterial

d) Apresenta real potencial para crescimento

3) As modificações introduzidas facilitam sua utilização e ampliam suas indicaçōes.

4) Os resultados obtidos até o momento definem a aorto-subclavioplastia com preservação do fluxo arterial como procedimento seguro e eficaz no tratamento da coarctação da procedimento seguro e eficaz no tratamento da coarctação da aorta. 


\section{RBCCV 44205-201}

MENDONÇA, J. T.; CARVALHO, M. R.; COSTA, R. K.; FRANCO FILHO, E.; COSTA, G. B. - Subclavian aortoplasty with preservation of arterial flow to the left upper limb: technical padronization. Rev. Bras. Cir. Cardiovasc., 8(2):63-82, 1993.

ABSTRACT: Between February 1984 and March 1992, 10 patients with aortic coarctation undetwent surgical correction with the technique of subclavian aortoplasty with preservation of arterial flow to the left upper limb. Eight of the patients were male and 2 were female. Their ages ranged from 2 months to 25 years (mean 6.3 years) and the weight ranged from 3.1 to $60 \mathrm{~kg}$ (mean $21.2 \mathrm{~kg}$ ). Surgical technique basically consisted of detachment of the left subclavian artery with subsequent reimplant as a ceiling over the constricted aortic area, thus preserving the arterial flow to the left arm. In this procedure two steps are fundamental: a) wide dissection of aorta and the left subclavian artery in order to allow full mobilization of those vessels, and b) anterior aortic incision should be large to allow reimplant of the subclavian proximal flap well beyond the constricted area. This will produce a wide anatomic reconstruction without tension on the suture lines. Two modifications of the original technique have been utilized: a) resection of a segment of anterior aortic wall the base of the subclavian artery. This will allow a good aortoplasty for patients with well formed isthmus with less dislodgement of the original subclavian insertion, and b) resection of the coarcted segment, end to end anastomosis of the posterior wall and anterior widening with the previously detached and posteriorly incised subclavian artery. This modification is best indicated for small children since it will remove the adjacent ductal tissue. The original technique was utilized for three patients; the modification with and anterior aortic flap was employed in 2 patients and the technique with simultaneous resection of the coarcted area was utilized in the other 5 patients. There was a single operative death, not related to the technique. Nine patients were followed for 1 to 8 years post-operatively (mean 3.9 years). All patients remained asymptomatic, with normal arterial blood pressure and normal pulses in the second day and six years (mean 1.6 years), and demonstrated an excellent aortic widening with preservation of arterial flow to the left arm in 8 patients. One patient had a small circular constriction which produced a $20 \mathrm{mmHg}$ gradient. In conclusion, the subclavian-aortoplasty with preservation of arterial flow to the left arm (basic or combined technique) allows an adequate anatomic correction, permits the subsequent aortic growth and can be utilized in the vast majority of cases of aortic coarctation treated during the pediatric age.

DESCRIPTORS: aortoplasty, subclavian; aortic coarctation, surgery.

\section{REFERÊNCIAS BIBLIOGRÁFICAS}

1 AMARAL, F. T. V. \& SOMERVILLE, J. - Coarctação da aorta: análise pós-operatória da pressão arterial em repouso. Arq. Bras. Cardiol., 46: 171-176, 1986.

AMATO, J. J.; GALDIERI, R. J.; COTRONEO, J. V. Role of extended aortoplasty related to the definition of coarctation of the aorta. Ann. Thorac. Surg., 52: 615-620, 1991.

3 AMATO, J. J.; RHEINLANDER, H. F.; CLEVELAND, R. J. - A method of enlarging the distal transverse arch in infants with hypoplasia and coarctation of the aorta. Ann. Thorac. Surg., 23: 261-263, 1977.

4 BARBERO-MARCIAL, M.; VERGINELLI, G.; SIRERA, J. C.; EBAID, M.; ZERBINI, E. J. - Surgical treatment of coarctation of results in 35 patients. J. Thorac. Cardiovasc. Surg., 30: 75-78, 1982.

5 BEEKMAN, R. M.; KATZ, B. P.; MOOREHEADSTEFFENS, C.; ROCCHINI, A. P. - Altered baroreceptor function in children with systolic hypertension after coarctation repair. Am. J. Cardiol., 52: 112113,1983
6 BEEKMAN, R. H.; ROCCHINI, A. P.; BEHRENDI, D. M.; BOVE, E. L.; DICK, I. I. M.; CROWLEY, D. C.; SNIDER, R.; ROSENTHAL, A. - Long-term outcome after repair of coarctation in infancy: subclavian angioplasty does not reduce the need for reoperation. J. Am. Coll. Cardiol., 8: 1406-1411, 1986.

7 BERGDAHL, L. \& LJUNGQVIST, A. - Long-term results after repair of coarctation of the aorta by patch grafting. J. Thorac. Cardiovasc. Surg., 80: 177 $181,1980$.

8 BERGDAHL, L. A. L.; BLACKSTONE, E. H.; KIRKLIN, J. W.; PACIFICO, A. D.; BARGERON Jr, L. M. Determinants of early success in repair of aortic coarctation in infants. J. Thorac. Cardiovasc. Surg. 83: 736-742, 1982.

9 BLALOCK, A. \& PARK, E. A. - Surgical treatment of experimental coarctation (atresia) of aorta. Ann. Surg., 119: 445-456, 1944.

10 BLALOCK, A. \& TAUSSIG, H. B. - The surgical treatment of malformations of the heart in which there is pulmonary stenosis or pulmonary atresia. JAMA, 128: $189-202,1945$ 
MENDONÇA, J.T.; CARVALHO, M.R.; COSTA, R.K.; FRANCO FILHO, E.; COSTA, G.B. - Aorto-subclavioplastia com preservação do fluxo arterial para o membro superior esquerdo. Rev. Bras. Cir. Cardiovasc., 8(2):63-82, 1993.

BROM, A. G. - Narrowing of the aortic isthmus and enlargment of the mind. J. Thorac. Cardiovasc., Surg., 50: 166-167, 1965.

12 BROMBERG, B. I.; BEEKMAN, R. H.; ROCCHINI, A. P. - Aortic aneurysm after patch aortoplasty repair of coarctacion: a prospective analysis of prevalence, screening tests and risks. J. Am. Coll. Cardiol., 14: 734-741, 1989.

13 BROUWER, M. H. J.; CROME-DIJKHUIS, A. H.; EBELS, T.; EIJGELAAR, A. - Growth of the hipoplastic aortic arch after simple coarctation resection and end-toend anastomosis. J. Thorac. Cardiovasc. Surg., 104: 426-433, 1992.

14 BROWN, J.; DUNN, J.M.; BRYMER, J.F.; KIRSH, M. M. - Simultaneous treatment of aortic stenosis and coarctation on left thoracotomy with apical aortic conduit. Ann. Thorac. Surg., 25: 364-367, 1978.

15 CAMPALANI, G.; FIRMIN, R. K.; VAUGHAN, M.; ROSS, D. N. - Surgical repair of coarctation of the aorta using the internal mammary artery as a free autogenous graft. J. Thorac. Cardiovasc. Surg., 90: 926-934, 1985.

16 CAMPBELL, D. B.; WALDHAUSEN, J. A.; PIERCE, W S.; FRIPP, R.; WHITMAN, V. - Should elective repair of coarctation of the aorta be done in infancy? J. Thorac. Cardiovasc. Surg., 88: 929-938, 1984.

17 CATANI, R.; De MIRA, A. S.; SALUM, P. M.; BENEDETTI, H.; MALUF, M. A.; BUFFOLO, E. - Nova técnica para correção de coarctação da aorta: aortoplastia com a artéria torácica interna pediculada. Arq. Bras. Cardiol., 59: (Supl. 2), 68, 1992 (Resumo).

CHRISTENSEN, N. A. - Coarctation of the aorta: hitorical review. Proc. Staff. Meet Mayo Clin., 23: 322-324, 1948.

COBANOGLU, A.; TEPLY, J. F.; GRUNKEMEIER, G. L.; SUNDERLAND, C. O.; STARR, A. - Coartation of the aorta in patients younger than three months: a critique of the subclavian flap operation. J. Thorac. Cardiovasc. Surg., 89: 128-135, 1985.

20 CONNOR, T. M. \& BAKER, W. P. - A comparison of coarctation resection and patch angioplasty using postexercise blood pressure measurements. Circulation, 64: 567-572, 1981.

21 CONNORS, J. P.; HARTMANN Jr., A. F.; WELDON, C. S. - Considerations in the surgical management of infantile coarctation of aorta. Am. J. Cardiol., 36: 489-492, 1975.

CRAFOORD, C. \& NYLIN, G. - Congenital coarctation of the aorta and its surgical treatment. J. Thorac. Surg., 14: 347-361, 1945. of the subclavian artery on the bones and soft tissues of the arms. J. Pediatr., 67: 808-811, 1965.
DEL NIDO, P. J.; WILLIANS, W. G.; WILSON, G. J. Synthetic patch angioplasty for repair of coarctation of the aorta: experience with aneurysm formation. Circulation, 74 (Supl. 1): 32-36, 1986.

DIETL, C. A. \& TORRES, A. R. - Coarctation of the aorta: anastomotic enlargement with subclavian artery: two new surgical options. Ann. Thorac. Surg., 43: 224-225, 1987.

DIETL, C. A.; TORRES, A. R.; CAZZANIGA, M. Coartectomia y anastomosis ampliada con subclavia: un procedimiento quirúgico modificado para el tratamiento de la coarctación en neonatos y lactentes. Rev. Latina Card. Inf., 1: 207-212, 1985.

27 DIETL, C. A.; TORRES, A. R.; FAVALORO, R.; FES SLER, C. L.; GRUNKEMEIR, A. - Risk of recoarctation in neonates and infants after repair with patch aortoplasty, subclavian flap, and the combined resection-flap procedure. J. Thorac. Cardiovasc. Surg., 103: 724-732, 1992.

ELLIOTT, M. J. - Coarctation of the aorta with arch hypoplasia: improvements on a new technique. Ann. Thorac. Surg., 44: 321-323, 1987.

ELZENGA, N. J. \& GITTENBERGER-DE GROOT, A. C. - Localized coarctation of the aorta: an age dependent spectrum. Br. Heart J., 49: 317-323, 1983.

ESCOBAR, M.; LIMA, R.; VICTOR, E.; TOMPSOM, G.; MORAES, C. R. - Cirurgia de coarctação da aorta na infância. Arq. Bras. Cardiol., 31 (Supl. 2), 158, 1978 (Resumo).

31 FISHMAN, N. H.; BRONSTEIN, M.H.; BERMAN Jr., W. ROE, B. B.; EDMUNDS Jr., L. H.; ROBINSON, S. T.; RUDOLPH, A. M. - Surgical management of severe aortic coarctation and interrupted aortic arch in neonates. J. Thorac. Cardiovasc. Surg., 71: 35-47, 1976.

32 FLEMING, W. H.; SARAFIN, J.B.; DOOLEY, K. J.; MOORING, P. K.; HOPEMAN, A. R. - Critical aortic coarctation: results of patch aortic coarctation: results of patch aortoplasty in infancy. Circulation, 55-56 (Supl. 3): 103, 1977 (Resumo)

33 FOLGER, G. M. \& SHAH, K. O. - Subclavian steal in patients with Blalock-Taussig anastomosis. Circulation, 31: 241-248, 1965.

34 FOURNIER, A.; CHARTRAD, C.; GUERIN, R.; DAVIGNON, A.; STANLEY, P. - Use of the internal mammary artery for preservation of circulation to the left arm coarctation in children. J. Thorac. Cardiovasc. Surg., 90: 926-934, 1985.

35 FRIPP, R. R.; WHITMAN, V.; WERNER, J. C.; NICHOLAS, G. C.; WALDHAUSEN, J. A. - Blood pressure response to exercise in children following the subclavian flap procedure for coarctation of the aorta. - J. Thorac. Cardiovasc. Surg., 85: 682-685, 1983. 
MENDONÇA, J.T.; CARVALHO, M.R.; COSTA, R.K.; FRANCO FILHO, E.; COSTA, G.B. - Aorto-subclavioplastia com preservação do fluxo arterial para o membro superior esquerdo. Rev. Bras. Cir. Cardiovasc., 8(2):63-82, 1993.

FLYER, D. C. - Report of the New England regional infant cardic program. Pediatrics., 65 (Supl.): 377 . $461,1980$.

37 GEISS, D.; WILLIAMS, W. G.; LINDSAY, W. K.; ROWE, R. D. - Upper extremity gangrene: a complication of subclavian artery division. Ann. Thorac. Surg., 30: $487-489,1980$.

GIDDING, S. S.; ROCCHINI, A. P.; MOOREHEAD, C.; SCHORK, M. A.; ROSENTHAL, A. - Increased forearm vascular reactivity in patients with hypertension after repair of coarctation. Circulation, 71: 495499, 1985.

GOMES, O. M.; PITCHOM, M.; CAETANO, M. C.; MORAIS, A. G.; BARROS, M. V. L.; VALENTE, R. R.; OLIVEIRA NETO, A. M. - Duplicação aórtica mediastinal para tratamento cirúrgico de recoarctação de aorta. Coração, 2: 12-14, 1990.

GONTIJO FILHO, B.; FANTINI, F. A.; SILVA, J. A. P.; BARBOSA, J. T.; SIMŌES, R.; FREIRE, R. J. A. Tratamento cirúrgico simultâneo de coarctação da aorta e insuficiência coronária: relato de caso. Arq. Bras. Cardiol., 41: 459-462, 1983.

GROSS, R. E. \& HUFNAGEL, C. A. - Coarctation of the aorta: experimental studies regarding surgical correction. N. Engl. J. Med., 233: 287-293, 1945.

GROSS, R. E.; HURWITT, E. S.; BILL, H. A.; PIERCE, E. C. - Preliminary observations on the use of human arterial grafts in the treatment of certain cardiovascular defects. New. Engl. J. Med., 239: 578-579, 1948.

HAMILTON, D. J.; EUSANIO, G. D.; SANDRASAGRA F. A.; DONNELY, R. J. - Early and late results of aortoplasty with a left subclavian flap for coarctation of the aorta in infancy. J. Thorac. Cardiovasc. Surg., 75: $699-704,1978$.

HAMMON, J. W.; GRAHAM, T. P.; BOUCEK, R. J.; BENDER, H. W. - Operative repair of coarctacion of the aorta in infancy: results with and without ventricular septal defect. Am. J. Cardiol., 55: 15551559, 1985.

HARLAN, J. L.; DOTY, D. B.; BRANDT, B.; EHRENHAFT, J. L. - Coarctation of the aorta in infants. J. Thorac. Cardiovasc. Surg., 88, 1012 . $1019,1984$.

HARTMANN Jr, A. F.; GOLDRING, D.; HERNAULEZ, A.; BEHRER, M. R.; SCHAD, N.; FERGUSON, T.; BURFORD, T. - Recurrent coarctation of the aorta after successful repair in infancy. Am. J. Cardiol., 25: 405-410, 1970.

47 HEHRLEIN, F. W.; MULCH, J.; RAUTENBERG, H. W.; SCHLEPPER, M.; SCHELD, H. H. - Incidence and pathogenesis of late aneurysms after patch graft aortoplasty for coarctation. J. Thorac. Cardiovasc. Surg., 92, 266-230, 1986.
48 HEIKKINEM, L. \& ALA-KULJU, K. - Long-term results of direct aortoplasty for repair of aortic coarctation in adults. Ann. Thorac. Surg., 49: 948-950, 1990.

49 HENZE, A. \& HALBERG, M. - The internal mammary artery free end flap aortoplasty in coarctation of the aorta. Scand. J. Thorac. Cardiovasc. Surg., 23: $39-42,1989$.

50 HENZE, A. \& HALBERG, M. - The internal mammary artery origin flap aortoplasty in coarctation of the aorta. Scand. J. Thorac. Cardiovasc. Surg., 23: 43-45, 1989.

51 HESSLEIN, P. S.; McNAMARA, D. G.; MORISS, M. J. H.; HALLMAN, G. L.; COOLEY, D. A. - Comparison of resection versus patch aortoplasty for repair of coarctation in infants and children. Circulation., 64: $164-168,1981$.

52 HO, S. Y. \& ANDERSON, R. H. - Coarctation, tubular hypoplasia and the ductus arteriosus: histological study of 35 specimens. Br. Heart J., 41: 268-274, 1979.

53 IGLER, F. O.; BOERBOOM, L. E.; WERNER, P. H. DONEGAN, J. H.; ZUPERLAU, E. J.; BONCHEK, L. I.; KAMPINE, K. P. - Coactation of the aorta and baroreceptores resecting. Circ. Res., 48: 365-371, 1981.

54 ISNER, J. M.; DONALDSON, R. F.; FULTON, D.; BHAN I.; PAYNE, D. D.; CLEVELAND, R. J. - Cistic medial necrosis in coarctation of the aorta: a potential factor contributing to adverse consequences observed after percutaneous balloon angioplasty of coarctation sites. Circulation., 75: 689-695, 1987.

55 JAMES, F. W. \& KAPLAN, S. - Systolic hypertension during submaximal exercise after correction of coarctation of aorta. Circulation, 49 (Supl. 2): 27 34,1973

56 JONAS, R. A. - Coarctation: do we need to resect ductal tissue? Ann. Thorac. Surg., 52: 604-607, 1991.

57 KALIL, R. A. K.; VITOLA, D.; CASTRO, I.; GUILLERMOCAL, L.; ZIELINSKY, P. E.; AZAMBUJA, P. C. Hipertensāo residual e teste de esforço no pós-operatório tardio de correção da coarctação da aorta. Arq. Bras. Cardiol., 44: 389-393, 1985.

$58 \mathrm{KEITH}$, J. D.; ROWE, R. D.; VLAD, P. (eds.) - Heart disease in infancy and childhood. New York, MacMillan. $1978.738 \mathrm{p}$.

59 KHOURY, G. H. \& HAWES, C. R. - Recurrent coarctation of the aorta in infancy and childhood. J. Pediatr., 72: $801-806,1968$

60 KIRKLIN \& BARRAT-BOYES (eds.) - Cardiac surgery. New York. John Wiley \& Sons, 1986. 1550 p.

61 KITTLE, C. F. \& SCHAFER, P. W. - Gangrene of the forearm after subclavian arterio-aortostomy for coarctation of the aorta. Thorax, 8: 319-321, 1953. 
MENDONÇA, J.T.; CARVALHO, M.R.; COSTA, R.K.; FRANCO FILHO, E.; COSTA, G.B. - Aorto-subclavioplastia com preservaçāo do fluxo arterial para o membro superior esquerdo. Rev. Bras. Cir. Cardiovasc., 8(2):63-82, 1993.

KORFER, R.; MEYER, H.; KLEIKAMP, G.; BIRCKS W. - Early and late results after resection and end-toend anastomosis of coarctation of the thoracic aorta in early infancy. J. Thorac. Cardiovasc. Surg., 89: 616-622, 1985.

63 KRON, I. L.; FLANAGAN, T. L.; RHEUBAN, K. S.; CARPENTER, M. A.; GUTGESELL, H. P.; BLACKBOURNE, L. H.; NOLAN, S. P. - Incidence and risk of reintervention after coarctation repair. Ann. Thorac Surg., 49: 920-926, 1990.

64 LACOUR-GAYET, T.; BRUNIAUX, J.; SERRAF, A.; CHAMBRAM, P.; BLAYSAT, G.; LOSAY, J.; PETIT, J.; KACHANER, J.; PLANCHE, C. - Hypoplastic transverse arch and coarctation in neonates. $J$. Thorac. Cardiovasc. Surg., 100: 808-816, 1990.

LADUSANS, E. J.; CAMPALANI, G. P.; QUERESHI, S. A.; OPIE, J.; BAKER, E. J.; TYNAN, M.; DEVERALL, P. B. - Recurrence of aortic coarctation following repair by re-implantation of the subclavian artery. Int. J. Cardiol., 23: 321-325, 1989.

LANSMAN, S.; SHAPIRO, A. J.; SCHILLER, M. S.; COOPER, R.; GALLA, J. D.; LOWERY, R. C.; GOLINKO, R.; ERGIN, M. A.; GRIEPP, R. B. - Extended aortic arch anastomosis for repair of coarctation in infancy. Circulation, 74 (Supl. 1): 37-41, 1986.

67 LAWLESS, C. E.; SAPSFORD, R. N.; PALLIS, C.; HALLIDIE-SMITH, K. A. - Ischemic injury to the brachial plexus following subclavian flap aortoplasty. J. Thorac. Cardiovasc. Surg., 84: 779-782, 1982.

68 LODGE, F. A.; LAMBERTI, J. J.; GOODMAN, A. H.; KIRPATRICK, S. E.; GEORGE, L.; MATHEWSON, J. W.; WALDMAN, J. D. - Vascular consequences of subclavian artery transection for the treatment of congenital heart disease. J. Thorac. Cardiovasc. Surg., 86: 18-23, 1983.

69 MARON, B. J.; HUMPHRIES, J. O.; ROWE, R. D.; MELLITS, E. D. - Prognosis of surgically corrected coarctation of the aorta: a 20-year postoperative appraisal. Circulation, 47: 119-126, 1973.

70 MAYER Jr., J. E. - Invited letter concerning: coarctation. J. Thorac. Cardiovasc. Surg., 101: 165-166, 1991.

71 MEARNS, J. A.; DEVERALL, P. B.; KESTER, R. C. Revascularization of an for incipient gangrene after Blalock-Taussig anastomosis. Br. J. Surg., 65: 467 $468,1978$.

72 MEIER, M. A. - Pathogenesis of aneurysm formation opposite prosthetic patches used for coarctation repair. J. Thorac. Cardiovasc. Surg., 94: 723-724, 1987 (Discussão).

MEIER, M. A.; LUCCHESE, F. A.; JAZBIK, W.; NESRALLA, I. A.; MENDONÇA, J. T. - A new technique for repair of aortic coarctation: subclavian flap aortoplasty with preservation of arterial blood flow to the

left arm. J. Thorac. Cardiovasc. Surg., 92: 1005$1012,1986$.

74

MEIER, M. A.; LUCCHESE, F. A.; JAZBIK, W.; ZIELINKY, P.; JASBIK, J. C.; NESRALLA, I. A.; COUTINHO, J. H. S. A.; ROSSI, M. B. - Morfologia da coarctação da aorta: implicações cirúrgicas. Arq. Bras. Cardiol., 48: (Supl. 1): 156, 1987.

MENDONÇA, J. T.; CARVALHO, M. R.; RIKA, R. K.; FRANCO, E. F. - Coarctação da aorta: um anova técnica cirúrgica. Arq. Bras. Cardiol., 44: 181-183, 1985.

MENDONÇA, J. T.; CARVALHO, M. R.; RIKA, R. K.; FRANCO, E. F. - Coarctation of the aorta: a new surgical technique. J. Thorac. Cardiovasc. Surg., 90: $445-447,1985$.

MESMER, B. J.; MINALE, C.; MUHLER, E.; BERNUTH, G. - Surgical correction of coarctation in early infancy: does surgical technique influence the result? Ann. Thorac. Surg., 52: 594-603, 1991.

78 METZDORFF, M.; COBANOGLU, A.; GRUNKEMEIER, G. L.; SUNDERLAND, C. O.; STARR, A. - Influence of age operation on late results with subclavian flap aortoplasty. J. Thorac. Cardiovasc. Surg., 89:241. $243,1981$.

MIDGLEY, F. M. \& McCLENATHAN, J. E. - Subclavian steal syndrome in the pediatric age group. Ann. Thorac. Surg., 24: 252-257, 1977.

MOOR, G. F.; IONESCU, M. I.; ROSS, D. N. - Surgical repair of coarctation of the aorta by patch grafting. Ann. Thorac. Surg., 14: 626-630, 1972.

81 MORAES, C. R.; ARRUDA, M. E.; LAGRECA Jr., R. Tratamento cirúrgico da coarctação da aorta por aortoplastia. Arq. Bras. Cardiol., 28: 171-174, 1975.

82 MORRIS, G. C.; COOLEY, D. A.; DeBAKEY, M. E.; CRAWFORD, E. S. - Coarctation of the aorta with particular emphasis upon improved techniques of surgical repair. J. Thorac. Cardiovasc. Surg., 40: 705-722, 1960.

83 MOULTON, A. L.; BRENNER, J. I.; ROBERTS, G.; TAVARES, S.; ALI, S.; NORDENBERT, A.; BURNS, J. E.; RINGEL, R.; BERMAN, M. A. - Subclavian flap repair of coarctation of the aorta in neonates: realization of growth potential. J. Thorac. Cardiovasc. Surg., 87: 220-235, 1984.

84 NANTON, M. A. \& OLLEY, P. M. - Residual hypertension after coarctectomy in children. Am. J. Cardiol., 37: 769-772, 1976

85 OLIVEIRA, S. A.; OLIVEIRA, H. A.; KEDOR, H. H.; AULER Jr., J. O.; SOUZA, J. M. - Variante técnica para operação de coarctação do arco aórtico. Arq. Bras. Cardiol., 37: 395-397, 1981.

86 OLSSON, P.; SONDERLUND, S.; DUBIEL, W. T.; 
MENDONÇA, J.T.; CARVALHO, M.R.; COSTA, R.K.; FRANCO FILHO, E.; COSTA, G.B. - Aorto-subclavioplastia com preservação do fluxo arterial para o membro superior esquerdo. Rev. Bras. Cir. Cardiovasc., 8(2):63-82, 1993.

OVENFORS, C. O. - Patch graft or tubular grafts in the repair of coarctation of the aorta: a follow-study. Scand. J. Thorac. Cardiovasc. Surg., 10: 139-143, 1976.

87 PAE Jr., W. E.; WYERS, J. L.; WALDHAUSEN, J. A.; PROPHET, G. A.; PIERCE, W. S. - Subclavian flap angioplasty: experimental study in growing piglets. J. Thorac. Cardiovasc. Surg., 82: 922-927, 1981.

88 PARKER, F. B.; FARREL, B.; STREETEN, D. H. P.; BLACKMAN, M. S.; SONDHEIMER, H. M.; ANDERSON Jr., G. H. - Hypertensive mechanisms in coarctation of the aorta. J. Thorac. Cardiovasc. Surg., 80: $568-673,1980$.

89 PATEL, R.; SINGH, S. P.; ABRAMS, L.; ROBERTS, K. D. - Coarctation of the aorta with special reference to infants: long-term results of operation in $126 \mathrm{ca}$ ses. Br. Heart J., 39: 1246-1253, 1977.

90 PELLEGRINO, A.; DEVERALL, P. B.; ANDERSON, R. H.; SMITH, A.; WILKINSON, J. L.; RUSSO, P.; TYNAN, M. - Aortic coarctation in the first three months of life: an anatomopathological study with respect to treatment. J. Thorac. Cardiovasc. Surg., 89: $121-127,1985$.

91 PENKOSKE, P. A.; WILLIAMS, W. G.; OLLEY, P. M.; LeBLANC, J.; TRUSLER, G. A.; MOES, C. A. F.; JUDAKIN, R.; ROWE, R. D. - Subclavian arterioplasty: repair of coarctation of the aorta in the first year of life. J. Thorac. Cardiovasc. Surg., 87: 894900, 1984.

92 PIERCE, W. S.; WALDHAUSEN, J. A.; BERMAN Jr., W.; WHITMAN, V. - Late results of the subclavian flap procedure in infants with coarctation of the thoracic aorta. Circulation, 58: (Supl. 1): 1-78, 1978.

93 FRATES, R. P.; LUCCHESE, F. A.; KALIL, R.; BERTOLETTI, V. E.; SANT'ANA, J. R. M.; DAUT, N.; NESRALA, I. A. - Correção da coarctação da aorta pela istmoplastia coma artéria subclavia esquerda em pacientes sintomáticos no primeiro ano de vida. Arq. Bras. Cardiol., 36: 403-406, 1981.

94 PRAXEDES, J. N.; MALHEIRO, P. S.; BARROS, R. T.; SILVA, H. B.; MARCONDES, M. - Hipertensão arterial por coarctação da aorta em cães: hemodinâmica renal e natriurese tensional. Arq. Bras. Cardiol., 46: 301-306, 1986.

95 REUL Jr., G. J.; KABBANI, S. S.; SANDIFORD, F. M.; WUKASCH, D. C.; COOLEY, D. A. - Repair of coarctation of the thoracic aorta by patch aortoplasty. J. Thorac. Cardiovasc. Surg., 68: 696-704, 1974.

96 RHEUBAN, K.; CARPENTER, M.; JEDEIKIN, R.; DAMMANN, F.; KRON, I.; WELLONS, J.; NOLAN, S. P. - Aortic aneurysm after patch angioplasty for coarctation in childhood. J. Am. Coll. Cardiol., 5: 476, 1985 (Resumo).

ROSSI, R. I.; HOROWITZ, E. S.; ZIELINSKY, P.;
NESRALLA, I. A.; LUCCHESE, F. A. - Cirurgia de Teles para coarctação da aorta: experiência do Instituto de Cardiologia do RS. Arq. Bras. Cardiol., 53 (Supl. 1) 19, 1989.

98 ROSSI, F.; RAUL, I.; ROSSI, M.; BERTOLETTI, J. C.; LUCCHESE, F. A.; ZIELINSKY, P.; MOURA, M.; FIRPO, C.; LEBOUTE, F.; AQUINO, F.; DAUDT, N. - Coarctaçāo da aorta: resultados cirúrgicos e acompanhamento tardio. Arq. Bras. Cardiol., 41 (Supl. 1): $43,1983$.

99 RUDOLPH, A. M. - The changes in the circulation after birth: their importance in congenital heart disease. Circulation, 41: 343-359, 1970.

100 RUDOLPH, A. M.; HEYMANN, M. A.; SPITZNAS, U. Haemodynamic considerations in the development of narrowing of the aorta. Am. J. Cardiol., 30: 514$525,1972$.

101 RUSSEL, G. A.; BERRY, P. J.; WATERSON, K.; DHASMANA, J. P.; WISCHEART, J. D. - Patterns of ductal tissue in coarctation of the aorta in the first three months of life. J. Thorac. Cardiovasc. Surg., 102: 596-601, 1991.

102 SADE, R. M.; CRAWFORD, F. A.; HOHN, A. R.; RIOPEL, D. A.; TAYLOR, A. B. - Growth of the aorta after prosthetic patch aortoplasty for coarctation in infants. Ann. Thorac. Surg., 38: 21-25, 1984.

103 SAMANEK, M.; GOETZOVA, J.; FIREZOVA, J.; SKOVRANEK, J. - Differences in muscle blood flow in upper and lower extremities of patients after correction coarctation of the aorta. Circulation., 54: 377-381, 1976.

104 SANCHEZ, G. R.; BALSARA, R. K.; DUNN, J. M.; MEHTA, A. V.; O'RIORDAN, A. C. - Recurrent obstruction after subclavian flap repair of coarctation of the aorta in infants: can it be predicted prevent ? J. Thorac. Cardiovasc. Surg., 91: 738-746, 1986.

105 SCIOLARO, C.; COPELAND, J.; CORK, R.; BARKENBUSH, M.; DONNERSTEIN, R.; GOLDBERG, S. - Long-term follow-up comparing subclavian flap angioplasty to resection with modified oblique endto-end anastomosis. J. Thorac. Cardiovasc. Surg., 101: 1-13, 1991.

106 SEALY, W. C. - Coarctation of the aorta and hypertension. Ann Thorac. Surg., 3: 15-28, 1967.

107 SEHESTED, J.; BAANDRUP, V.; MIKKELSEN, E. - Different reativity and structure of the prestenotic and poststenotic aortic in human coarctation. Circulation, 65: 1060-1064, 1982.

108 SELLORS, T. H. \& HOBSLEY, M. - Coarctation of the aorta: effect of operation on blood pressure. Lancet, 1: 1387-1391, 1963.

109 SHARMA, B. J.; CALDERON, M.; OTT, D. A. - Coarctation reapair in neonates with subclavian sparing 
MENDONÇA, J.T.; CARVALHO, M.R.; COSTA, R.K.; FRANCO FILHO, E.; COSTA, G.B. - Aorto-subclavioplastia com preservação do fluxo arterial para o membro superior esquerdo. Rev. Bras. Cir. Cardiovasc., 8(2):63-82, 1993.

advanced flap. Ann. Thorac. Surg., 54: 137-141, 1992.

110 SHEARER, W. T.; RUTMAN, J. Y.; WEINBERG, W. A.; GODRING, D. - Coarctation of the aorta and cerebrovascular accident: a proposal for early corrective surgery. J. Pediatr., 77: 1004-1009, 1970.

111 SHINEBOURNE, E. A.; TAM, A. S. Y.; ELSEED, A. M.; PENETH, M.; LENNOX, S. C.; CLEAND, W. P.; LINCOLN, C.; JOSEPH, M. C.; ANDERSON, R. H. - Coarctation of the aorta in infancy and childhood. Br. Heart. J., 38: 375-380, 1976.

112 SIEWERS, R. D.; ETTEDGUI, J.; PAHL, E.; TALLMAN, T.; DEL NIDO, P. J. - Coarctation and hypoplasia of the aortic arch: will the arch grow? Ann. Thorac. Surg., 52: 608-614, 1991.

113 SILVER, M. M.; FREEDOM, R. M.; SILVER, M. D.; OLLEY, P. M. - The morphology of the newborn ductus arteriosus. Hum. Pathol., 12: 1123-1136, 1981.

114 SKOVRANEK, J.; GOETZOVA, J.; SAMANEK, M. Changes in muscle blood flow and development of the arm following the Blalock-Taussig anastomosis. Cardiology., 6: 131-137, 1975.

115 SWEENEY, M. S.; WALKER, W. E.; DUNCAN, J. M.; HALLMAN, G. L.; LIVESAY, J. J.; COOLEY, D. A. - Reoperation for aortic various approaches. Ann. Thorac. Surg., 40: 46-49, 1985.

116 TAWES, R. L.; ABERDEEN, E.; WATERSTON, D. J.; BONHAN-CARTER, R. E. - Coarctation of the aorta in infants and children: a review of 333 operative cases including 170 infants. Circulation, 39-40 (Supl. 1): 173-184, 1969.

117 TAYLOR, S. H. \& DONALD, K. W. - Circulatory studies at rest and during exercise in coarctation of the aorta before and after operation. Br. Heart. J., 22: 117. 139,1960

118 TODD, P. J.; DANGERFIELD, P. H.; HAMILTON, D. I.; WILKINSON, J. L. - Late effects on the left upper limb of subclavian flap aortoplasty. J. Thorac. Cardiovasc. Surg., 85: 678-681, 1983.

119 TRINQUET, F.; VOUHE, P.; VERNANT, F. - Coarctation of the aorta in infants: which operation? Ann. Thorac. Surg., 45: 186-191, 1988.

120 VAN SOM, J. A. M.; DANIELS, O.; VINCENT, J. G.; VANLIER, H. J. J.; LACQUET, L. K. - Appraisal of resection and end-to-end anastomosis for repair of coarctation of the aorta in infancy: preference for resection. Ann. Thorac. Surg., 48: 496-502, 1989.

121 VAN SON, J. A. M.; VAN ASTEN, W. N. J. C.; VAN LIER, H. J. J. - Detrimental sequelae on the hemodynamics of the upper left limb after subclavian flap angioplasty in infancy. Circulation. 81: 9961004,1990
122 VAN SON, J. A. M.; VAN ASTEN, W. N. J. C.; VAN LIER, H. J. J.; DANIELS, O.; SKOTNICKI, S. H.; LACQUET, L. K. - A comparison of coarctation resection and subclavia flapangioplasty using ultrasonographically monitored postocclusive reative hyperemia. $J$. Thorac. Cardiovasc. Surg., 100: 817-829, 1970.

123 VAN RIJK-ZWIKKER, G. L. - The efect of polidioxane and prolene on circular aortic sutures under tension. Net. Tiidschr. Geneeskd., 130: 801-802, 1986.

124 VERSCHUERE, I.; FRANÇOIS, K.; DeROOSE, J.; BERZSENYI, G.; DERMON, F. - Polidioxanone suture material in growing vascular anastomoses: experimental study. J. Thorac. Cardiovasc. Surg., 90: 765-770, 1985.

125 VERSKA, J. J.; DeQUATRO, V.; WOOLLEY, M. M. Coarctation of the aorta: the abdominal pain syndrome and paradoxical hypertension. J. Thorac. Cardiovasc. Surg., 58: 746-753, 1969.

126 VINCENT, J. G.; DANIELS, O.; VAN OORT, A.; LACQUET, L. K. - Hypoplastic aortic arch with aortic coarctation: surgical correction. J. Thorac. Cardiovasc. Surg., 89: 465-468, 1985.

127 VOSSCHULTE, K., - Istmusplastik zur behandlund der aortomisthmusstenose. Thoraxchirurgie, 4: 443. 447,1957

128 VOSSCHULTE, K. - Surgical corretion of the aorta by an "isthmusplastic" operation. Thorax, 16: 338-345, 1961.

129 VOUHE, P. R.; TRINQUET, F.; LECOMPTE, Y.; VERNANT, F.; ROUX, P. M.; TOUATI, G.; POME, G.; LECA, F.; NEVEUX, J. Y. - Aortic coarctation with hypoplastic aortic arch: results of extended end-toend aortic arch anastomosis. J. Thorac. Cardiovasc. Surg., 96: 557-563, 1988.

130 WALDHAUSEN, J. A. \& NAHRWOLD, D. L. - Repair of coarctation of the aorta with a subclavian flap. J. Thorac. Cardiovasc. Surg., 51: 532-533, 1966.

131 WALDMAN, J. D.; GOODMAN, A. H.; TUMEO, A. R.; LAMBERTI, J. J.; TURNER, S. W. - Coarctation of the aorta: noninasive physiological assessment in infants and children before and after operation. J. Thorac. Cardiovasc. Surg., 80: 187-197, 1980.

132 WALDMAN, J. D.; LAMBERTI, J. J.; GOODMAN, A. H.; MATHEWSON, J. W.; KIRKPATRICK, S. E.; GEORGE, L.; TURNER, S. W.; PAPPELBAUM, S. Coarctation in the first year of life. J. Thorac. Cardiovasc. Surg., 86: 9-17, 1983.

133 WEBB, W. R. \& BURFORD, T. H. - Gangrene of the arm following use of the subclavian artery in pulmonosystemic (Blalock) anastomosis. J. Thorac. Cardiovasc. Surg., 23: 199-204, 1952.

134 WELDON, C. S.; HARTMANN, A. F.; STEINHOFF, N. G.; MORRISSEY, J. D. - A simple, safe, and rapid 
MENDONÇA, J.T.; CARVALHO, M.R.; COSTA, R.K.; FRANCO FILHO, E.; COSTA, G.B. - Aorto-subclavioplastia com preservação do fluxo arterial para o membro superior esquerdo. Rev. Bras. Cir. Cardiovasc., 8(2):63-82, 1993.

technique for the management of recurrent coarctation of the aorta. Ann. Thorac. Surg., 15: 510-519, 1973.

135 WILLIAMS, W. G.; SHINDO, G.; TRUSLER, G. A.; DISCHE, M. R.; OLLEY, P. M. - Results of repair of coarctation of the aorta during infancy. J. Thorac. Cardiovasc. Surg., 79: 603-608, 1980.

136 YEE, E. S.; TURLEY, K.; SOIFER, S.; EBERT, P. A. Synthetic patch aortoplasty: a simplified approach for coarctation in repairs during early infancy and thereafter. Am. J. Surg., 148: 240-243, 1984.

137 ZANINE, L.; LECOMPTE, Y.; GALLI, R. - Aortic coarctation with arch hypoplasia: a new surgical technique. G. Ital. Cardiol., 15: 1045-1048, 1985.

138 ZIEMER, G.; JONAS, R. A.; PERRY, S. B.; FREED, M. D.; CASTANEDA, A. R. - Surgery for coarctation on the aorta in the neonates. Circulation., 74 (Supl. 1): 25-31, 1986 .

\section{Discussão}

\section{DR. RENATO A. K. KALIL \\ Porto Alegre, RS}

A técnica relatada por Teles de Mendonça, para correção de coarctação da aorta representa uma significativa contribuição da cirurgia cardiaca brasileira à especialidade. Tem sido reconhecida internacionalmente como alternativa válida e empregada em muitos centros. À maior complexidade técnica contrapõem-se as vantagens da preservação do fluxo arterial ao membro superior e a ausência de sutura circunferencial na aorta. Serão estas vantagens, no entanto, significativas? Complicações graves pela ligadura da artéria subclávia são extremamente raras e a experiência tem demonstrado que a simples presença de sutura circunferencial na aorta com fio de Polipropileno não é suficiente para impedir seu crescimento. Por outro lado, existem fios reabsorvíveis, de polidioxanona, para esta finalidade. $O$ trabalho apresentado tem o mérito de padronizar a técnica, mostrando os detalhes cuja observância é indispensável para o sucesso do procedimento. Estas informações são da maior importância. Na causística, notamos que a média de idade é 6,3 anos, sendo apenas 4 os pacientes menores de 1 ano de idade. Os resultados imediatos foram excelentes; entretanto, a maioria das angiografias foi realizada cerca de um ano após a cirurgia. Dos 4 pacientes menores de um ano, 3 realizaram angiofrafia e 1 apresenta estenose residual. O restante foi o óbito da série. Na experiência do IC/FUC, entre janeiro 1984 e setembro 1991 , foram operados 24 pacientes com menos de 1 ano de idade, de coarctação da aorta, pela técnica de Teles. Seis apresentavam lesões intracardíacas associadas. Ocorreram 3 óbitos hospi- talares $(12,5 \%)$, de causas aparentemente não relacionadas à técnica. No acompanhamento tardio, temos 12 pacientes assintomáticos, 3 foram submetidos a angioplastia por recoarctação, 1 apresenta estenose residual mínima, 1 foi a óbito após correção de CIV e 4 estão sem acompanhamento. Nossa conduta atual tem sido a de realizar ressecção do segmento coarctado e do tecido do canal arterial, seguida de anastomose término-terminal. Reservamos as técnicas de Waldhausen e de Teles para situações especiais. Gostaríamos de colocar aos autores as seguintes questões: 1) Qual vantagem haveria no emprego da técnica em crianças maiores e adultos, sobre a anastomose términoterminal, ou mesmo a interposição de tubo? 2) Em quatros casos a técnica foi tentada e não foi possível realizar? 3) Nos casos de ressecção, anastomose término-terminal e aorto-subclavioplastia realizadas em lactentes, as vantagens teóricas justificariam a maior complexidade técnica? Para finalizar, cumprimentamos o Dr. Teles pelo brilhantismo da idéia, por seus bons resultados e pela bela apresentação, ao mesmo tempo em que agradecemos aos autores o envio do trabalho completo para apreciação, agradecendo, também, à Comissão Organizadora o privilégio do convite para comentá-lo.

\section{DR. TELES DE MENDONÇA (Encerrando)}

Agradeço ao Dr. Kalil os comentários gentís e bem estruturados a respeito do que acabamos de apresentar. Procurarei ser breve e bastante objetivo na resposta às suas indagações. A cirurgia de coarctação da aorta, como foi dito na apresentação, deve obedecer aos seguintes princípios: a) promover completo alívio da obstrução; b) não utilizar nenhum material estranho ao organismo; c) não sacrificar parte da árvore arterial; d) apresentar real potencial para crescimento. Nos lactentes deve-se, ainda, acrescentar a remoção do seguimento coarctado, uma vez que ele mantém restos de tecido ductal, grande responsável pelos elevados índices de recoarctação, quando a operação é feita nesta idade. Portanto, nas crianças maiores e adultos, quando possível, a ressecção com anastomose término-terminal nos parece um bom procedimento. Nos lactentes, no entanto, a ressecção com anastomose término-terminal posterior associada com a ampliação anterior com a artéria subclávia, como sugerimos, nos parece ser a cirurgia ideal. Nas crianças a mobilização dos vasos é extremamente fácil, o que torna o procedimento muito simples, sendo sua realização possível na maioria dos casos operados. Em nenhum caso de nossa série o procedimento foi tentado e não exe- 
MENDONÇA, J.T.; CARVALHO, M.R.; COSTA, R.K.; FRANCO FILHO, E.; COSTA, G.B. - Aorto-subclavioplastia com preservação do fluxo arterial para o membro superior esquerdo. Rev. Bras. Cir. Cardiovasc., 8(2):63-82, 1993.

cutado. Finalizando, acredito que os benefícios reais conseguidos com a associação de anastomose término-terminal e aorto-subclavioplastia, em lactentes, possam justificar plenamente a maior complecidade e o emprego sistemático desta técnica. 